NIST Technical Note 2053

\title{
Users Guide to Type277 Loosely-Coupled Integration of TRNSYS with Java
}

Farhad Omar

This publication is available free of charge from: https://doi.org/10.6028/NIST.TN.2053

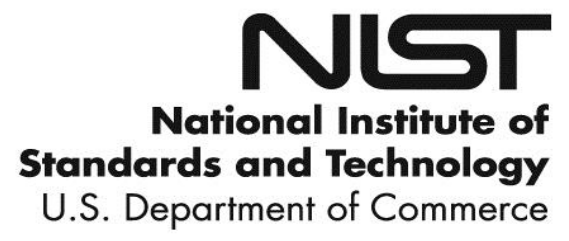




\title{
Users Guide to Type277 Loosely-Coupled Integration of TRNSYS with Java
}

\author{
Farhad Omar \\ Energy and Environment Division \\ Engineering Laboratory
}

This publication is available free of charge from:

https://doi.org/10.6028/NIST.TN.2053

August 2019

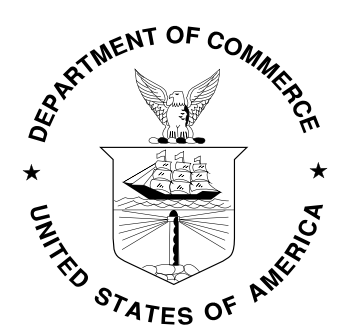

U.S. Department of Commerce

Wilbur L. Ross, Jr., Secretary

National Institute of Standards and Technology

Walter Copan, NIST Director and Undersecretary of Commerce for Standards and Technology 
Certain commercial entities, equipment, or materials may be identified in this document in order to describe an experimental procedure or concept adequately. Such identification is not intended to imply recommendation or endorsement by the National Institute of Standards and Technology, nor is it intended to imply that the entities, materials, or equipment are necessarily the best available for the purpose.

National Institute of Standards and Technology Technical Note 2053

Natl. Inst. Stand. Technol. Tech. Note 2053, 34 pages (August 2019) CODEN: NTNOEF

This publication is available free of charge from: https://doi.org/10.6028/NIST.TN.2053 


\section{Software Disclaimers}

Any mention of commercial products in Type277 and this user's guide is for information purposes only; it does not imply recommendation or endorsement by NIST.

This software was developed by employees of the National Institute of Standards and Technology (NIST), an agency of the Federal Government and is being made available as a public service. Pursuant to Title 17 United States Code Section 105, works of NIST employees are not subject to copyright protection in the United States. This software may be subject to foreign copyright. Permission in the United States and in foreign countries, to the extent that NIST may hold copyright, to use, copy, modify, create derivative works, and distribute this software and its documentation without fee is hereby granted on a nonexclusive basis, provided that this notice and disclaimer of warranty appears in all copies.

THE SOFTWARE IS PROVIDED 'AS IS' WITHOUT ANY WARRANTY OF ANY KIND, EITHER EXPRESSED, IMPLIED, OR STATUTORY, INCLUDING, BUT NOT LIMITED TO, ANY WARRANTY THAT THE SOFTWARE WILL CONFORM TO SPECIFICATIONS, ANY IMPLIED WARRANTIES OF MERCHANTABILITY, FITNESS FOR A PARTICULAR PURPOSE, AND FREEDOM FROM INFRINGEMENT, AND ANY WARRANTY THAT THE DOCUMENTATION WILL CONFORM TO THE SOFTWARE, OR ANY WARRANTY THAT THE SOFTWARE WILL BE ERROR FREE. IN NO EVENT SHALL NIST BE LIABLE FOR ANY DAMAGES, INCLUDING, BUT NOT LIMITED TO, DIRECT, INDIRECT, SPECIAL OR CONSEQUENTIAL DAMAGES, ARISING OUT OF, RESULTING FROM, OR IN ANY WAY CONNECTED WITH THIS SOFTWARE, WHETHER OR NOT BASED UPON WARRANTY, CONTRACT, TORT, OR OTHERWISE, WHETHER OR NOT INJURY WAS SUSTAINED BY PERSONS OR PROPERTY OR OTHERWISE, AND WHETHER OR NOT LOSS WAS SUSTAINED FROM, OR AROSE OUT OF THE RESULTS OF, OR USE OF, THE SOFTWARE OR SERVICES PROVIDED HEREUNDER.

\section{Copyright Notice for External Libraries}

The JTRNSYS project includes external libraries such as Protocol Buffer, JavaFX, and matlabcontrol, please read/adhere to the license agreements of these libraries reproduced in the $3^{\mathrm{RD}}$ PPARTY_LICENSEs.txt file. 


\section{Table of Contents}

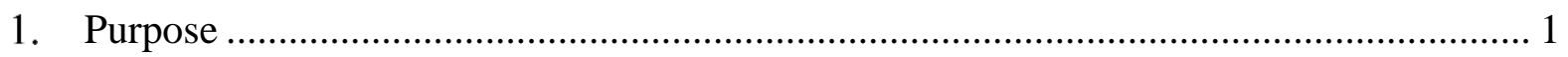

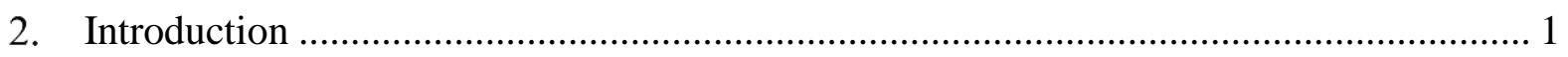

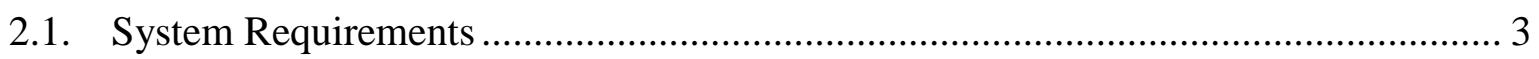

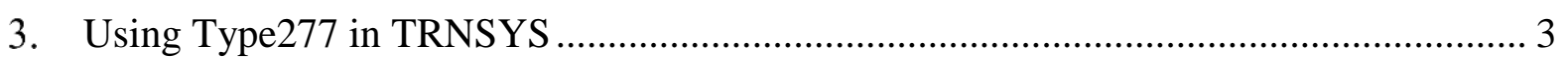

3.1. Downloading JTRNSYS Project ................................................................. 5

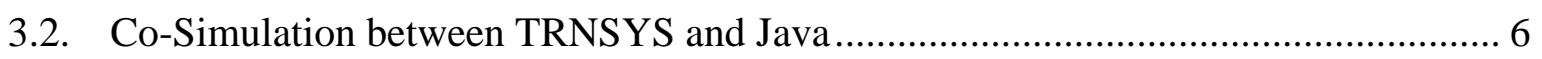

3.2.1. Simulating the PVT Model in TRNSYS ..................................................... 7

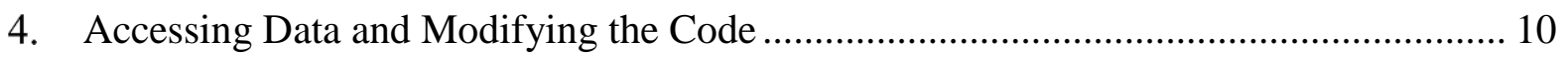

4.1. ServerRunnable Class......................................................................................... 11

4.2. ServerRunnableMatlab Class ............................................................................ 13

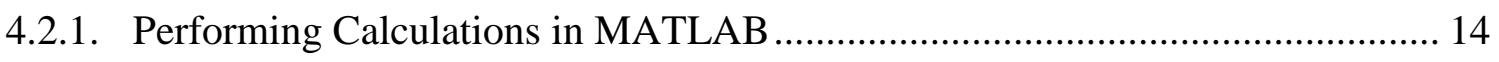

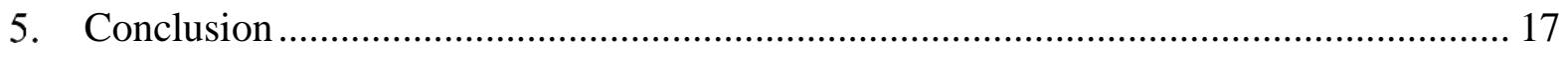

Appendix - Importing JTRNSYS Project into Eclipse............................................... 18

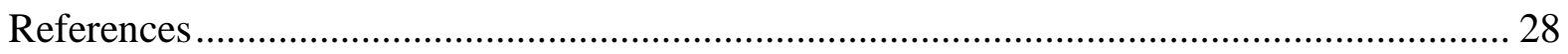




\section{List of Figures}

Figure 1. A schematic representation of the co-simulation environment .............................. 1

Figure 2. A UML class diagram of the JAVA classes used for implementing a local server

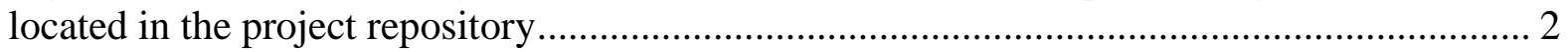

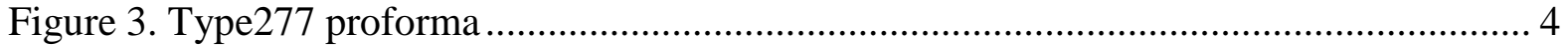

Figure 4. The PVT example model in TRNSYS [1] ................................................. 5

Figure 5. User interface for starting the server ....................................................... 6

Figure 6. Open the PVTType277 model ...................................................................... 8

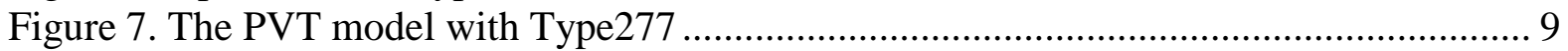

Figure 8. Create a TRNSYS input file for PVTType277 model........................................ 10

Figure 9. Result of simulating the PVTType277 example with the local server in Java....... 10

Figure 10. Create an object of ServerRunnable class for launching the server (see

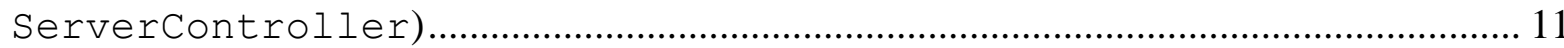

Figure 11. Code snippet for processing incoming data from TRNSYS (see

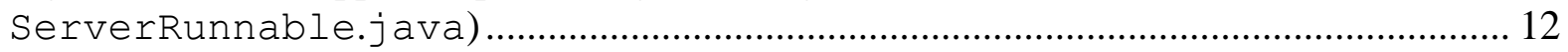

Figure 12. Access data from TRNSYS (see ServerRunnable. java).......................... 12

Figure 13. Sending serialized data back to TRNSYS (see ServerRunnable. java)..... 12 Figure 14. UML class diagram for loosely-coupling TRNSYS and MATLAB via the Java

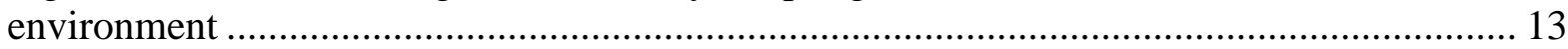

Figure 15. Create an object ServerRunnableMATLAB for exchanging data between

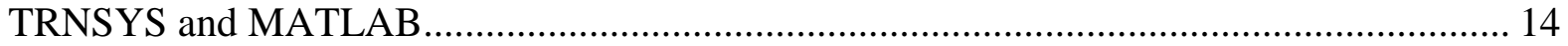

Figure 16. Adding Matlab Files folder to the path of MATLAB software........................... 15

Figure 17. Methods for facilitating the exchange of data between TRNSYS and MATLAB 15

Figure 18. Proxy function call to send and receive data to-and-from MATLAB ................. 16

Figure 19. Method to serialize and return data to TRNSYS ........................................... 16

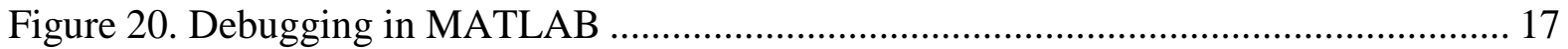

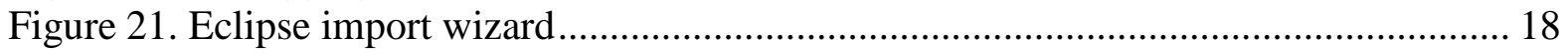

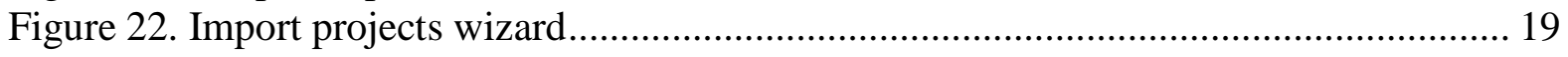

Figure 23. Project explorer in Eclipse........................................................................... 20

Figure 24. Opening properties window for JTRNSYS .............................................. 21

Figure 25. Editing Java build path for the missing libraries .............................................. 22

Figure 26. Adding third-party libraries to the build path for JTRNSYS project .................. 22

Figure 27. Adding a user library to the JTRNSYS project .............................................. 23

Figure 28. Adding a new JavaFX 11 user library to the JTRNSYS project ........................ 24

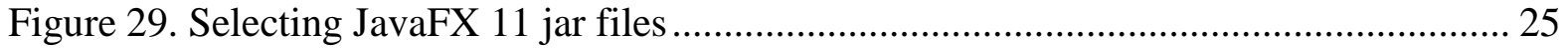

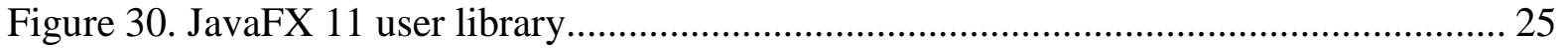

Figure 31. Close the Add Library window ........................................................................ 26

Figure 32. Added external libraries to the build path of the JTRNSYS project ................... 26 


\section{Purpose}

The purpose of this document is to provide instructions for using a newly developed TRNSYS component (Type277). Type277 utilizes socket communication to provide loosely-coupled integration between TRNSYS and Java-based components. Bi-directional flow of information is facilitated using the client-server architecture of Java. Type277 is compiled as both a 32-bit and 64-bit dynamic link library to support co-simulation with TRNSYS17 and TRNSYS18 projects. An application of Type 277 is demonstrated by modifying an example TRNSYS project. The Java project and files, TRNSYS modified example projects, and Type 277 dynamic link libraries are being made available to the public through the NIST GitHub site.

\section{Introduction}

This section describes the use of NIST-developed software (Type277) for performing cosimulation by facilitating the exchange of data between a local server and a model developed with the TRaNsient SYstem Simulation tool (TRNSYS) [1]. A detailed description of the cosimulation environment is provided in [2]. Figure 1 shows a schematic representation of the co-simulation environment, describing the interaction of a TRNSYS model and a local server. Figure 1 also shows that Type277 enables a TRNSYS model to participate in a co-simulation with a third-party software tool through the local server. An example of co-simulation between TRNSYS and MATLAB is described in Section 4.2. The connection between TRNSYS and the local server is accomplished through socket communication, while the connection between the local server and the third-party software can be established using proxies or other available libraries.

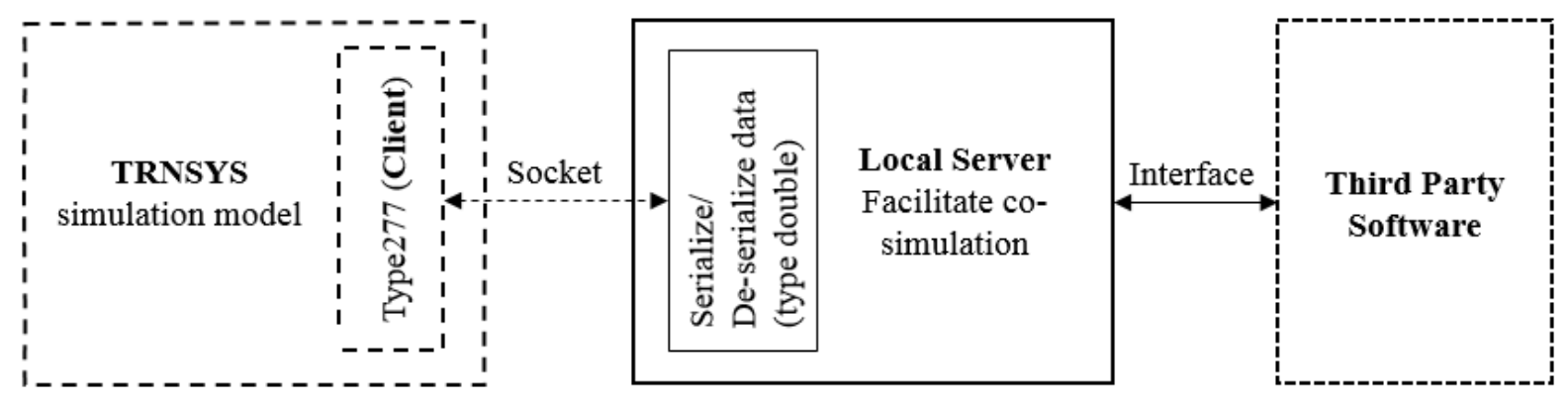

Figure 1. A schematic representation of the co-simulation environment

The co-simulation environment shown in Figure 1 was implemented in a Java project, which includes all the necessary folders and files used for loosely-coupled integration of TRNSYS with a Java environment. The key idea behind this approach is to demonstrate the use of Type277 and enable users to modify the code to meet their needs. Figure 2 shows a Unified Modelling Language (UML) class diagram of the Java project without the interface to a thirdparty software tool. 


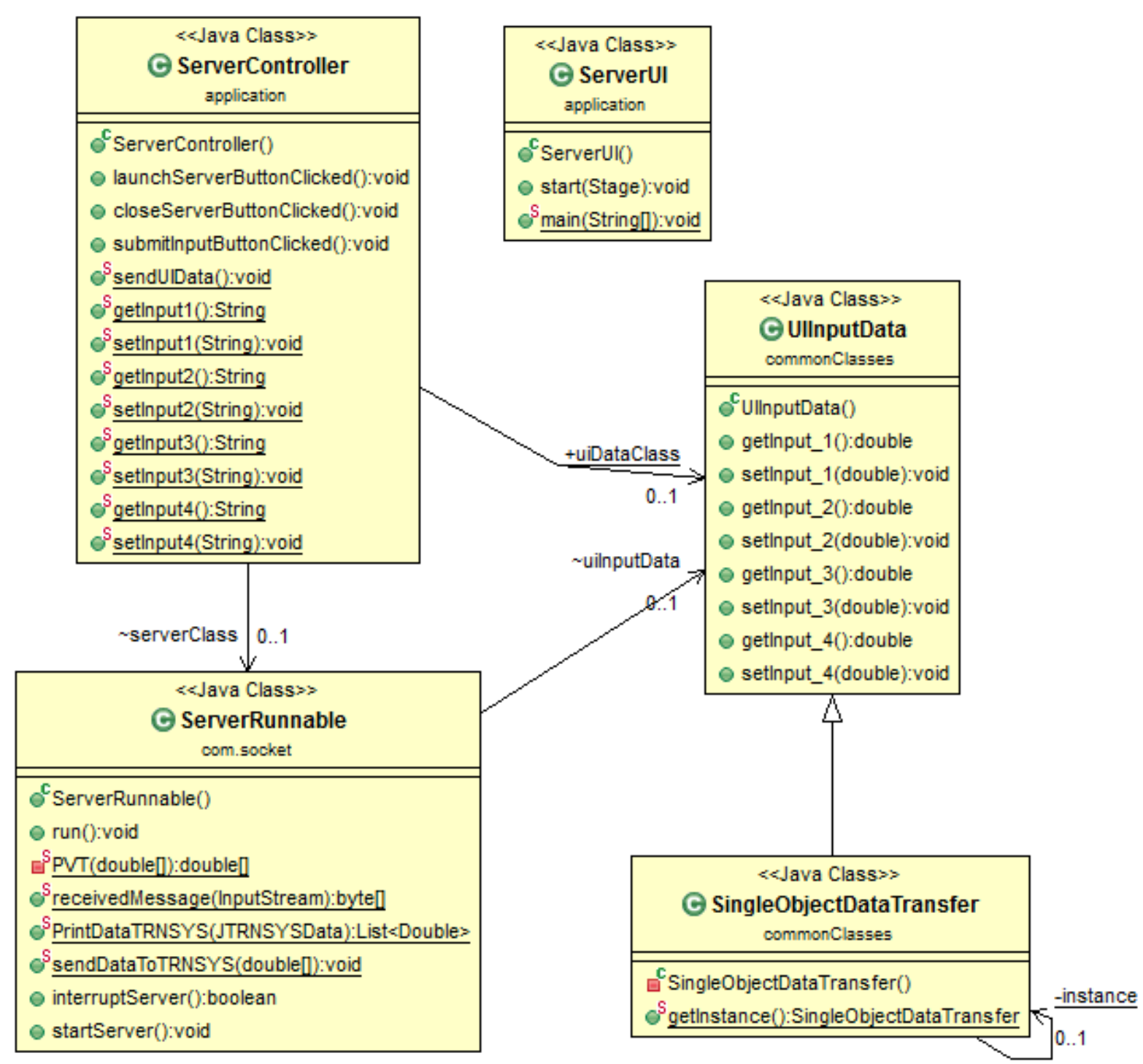

Figure 2. A UML class diagram of the JAVA classes used for implementing a local server located in the project repository

The UML class diagram shown in Figure 2, describes the relationship of various classes used to support the loosely-coupled integration of TRNSYS and Java. The ServerUI is the main class for rendering the graphical user interface (GUI) and creating an instance of the controller class (ServerController). The ServerController class controls the functionality of the GUI and instantiates an object of the ServerRunnable class, which implements the server and facilitates the exchange of data between TRNSYS and Java. The instructions for accessing TRNSYS data in ServerRunnable class are presented in Section 4.1. The ServerController class also instantiates an object of UI InputData class to capture the user inputs (e.g., get Input_1 ( ) shown in Figure 2) from the GUI and transfer them to other classes using a Singleton object. 


\subsection{System Requirements}

1. Personal computer able to run Microsoft Windows 7 or 10 versions;

2. Java Standard Edition (SE) 8 for Windows (JRE 1.8) or Java Development Kit (JDK 1.8);

3. JavaFX 11 libraries (included in the project, see Appendix) or earlier versions;

4. MATLAB Java application programming interface matlabcontrol-4.1.0.jar (included in the project, see Appendix); and

5. Google's Protocol Buffer library protobuf-java-3.6.1.jar (included in the project, see Appendix).

Note that an implementation of protocol buffers used in this project is not compatible with JDK 11. This project was created and tested using JRE 1.8 or JDK 1.8 for Windows. Also, note that MATLAB software must be available in your system to use the example described in Section 4.2.

\section{Using Type277 in TRNSYS}

TRNSYS is a modular and extendable simulation environment that consists of a suite of software tools designed to accommodate transient simulation of multi-zone buildings and other thermal systems. The main user interface is Simulation Studio, in which users can setup projects by graphically connecting model components. Each component is mathematically described in the TRNSYS simulation engine and has a corresponding graphical representation (proforma) in Simulation Studio. A proforma is a black-box description of inputs, outputs, and parameters. TRNSYS components are commonly referred to as Types and are identified by a number which relates a component to the model of that component written as a subroutine. An advantage of the TRNSYS modular architecture is its ability to support the integration of userdefined types, such as Type277.

Type277 is a dynamic-linked library (DLL) designed to facilitate the loosely-coupled integration of a simulation model in TRNSYS with components written in other programming languages, e.g., Java and MATLAB. Type277 utilizes socket communication to establish a connection between TRNSYS and a local server; effectively converting a TRNSYS model into a client of the server. To ensure a reliable exchange of information between a server and the client, the data of the primitive type Double are serialized using Google's protocol buffers [3]. Type277 is written in C++ and compiled into 32-bit and 64-bit DLLs. This document presents a step-by-step instruction for using Type277 in a TRNSYS model while exchanging data with a server implemented in Java. Alternative implementation of the local server in C++ and Python are also made possible by serializing data with protocol buffers. Relevant C++ and Python libraries are included with the Java project download from NIST's GitHub site.

Like all standard types in TRNSYS, Type277 has a proforma as shown in Figure 3. As can be seen from Figure 3, the value associated with the first parameter sets the number of inputs and the value associated with the second parameter sets the number of outputs. Type 277 uses these parameters to set the size of a vector ( $1 \mathrm{x} \mathrm{n}$ dimensional array) for exchanging data of the 
primitive type Double. The input data are sent to the server and the returned values can be connected to the Inputs of other types in a TRNSYS project.

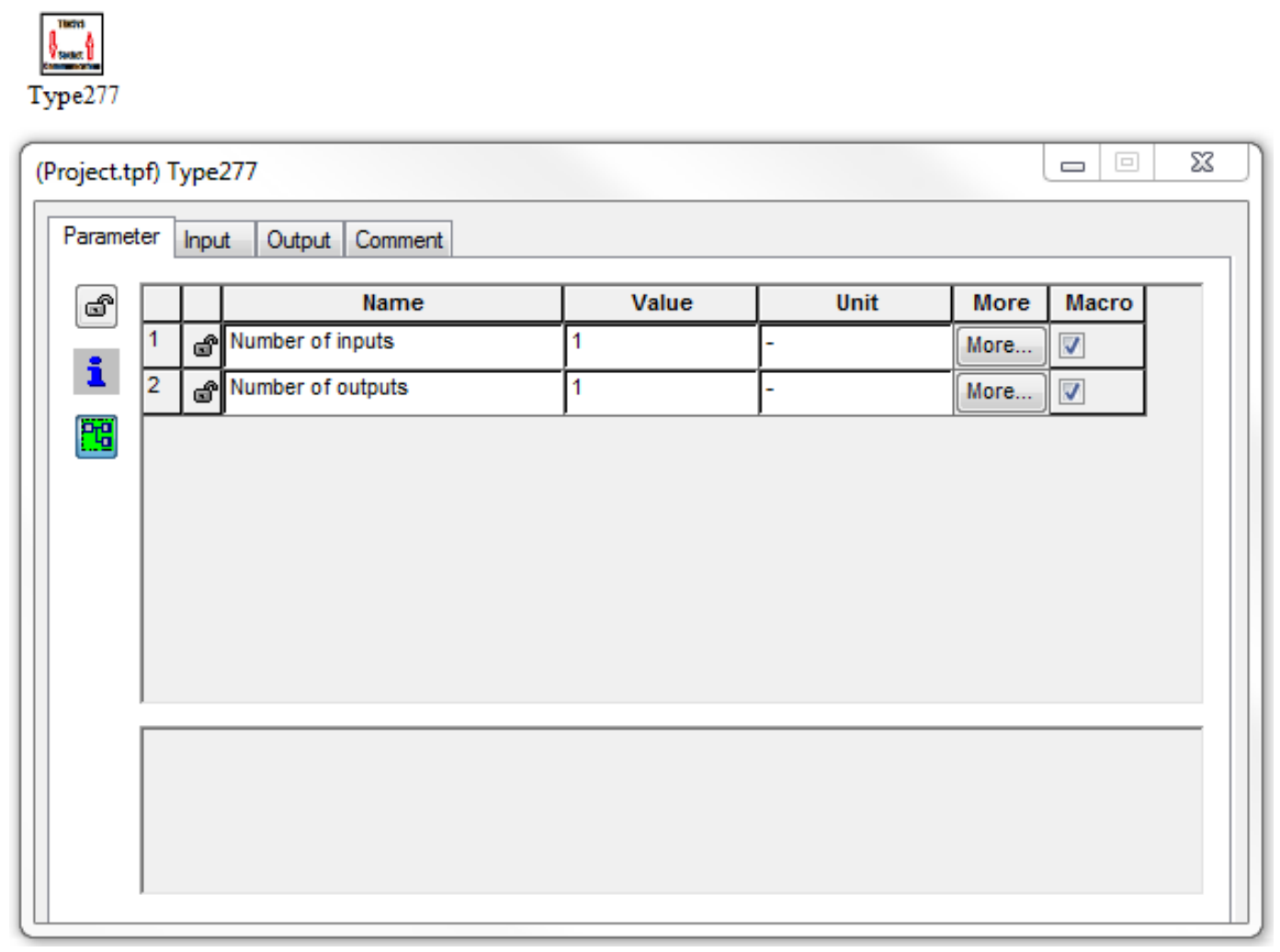

Figure 3. Type 277 proforma

To demonstrate the use of Type277 in a TRNSYS model, the PVT example TRNSYS project was modified by replacing the pLoad Equation shown in Figure 4 with Type277. The goal is to calculate pLoad in Java and MATLAB and return the calculated values back to TRNSYS. 


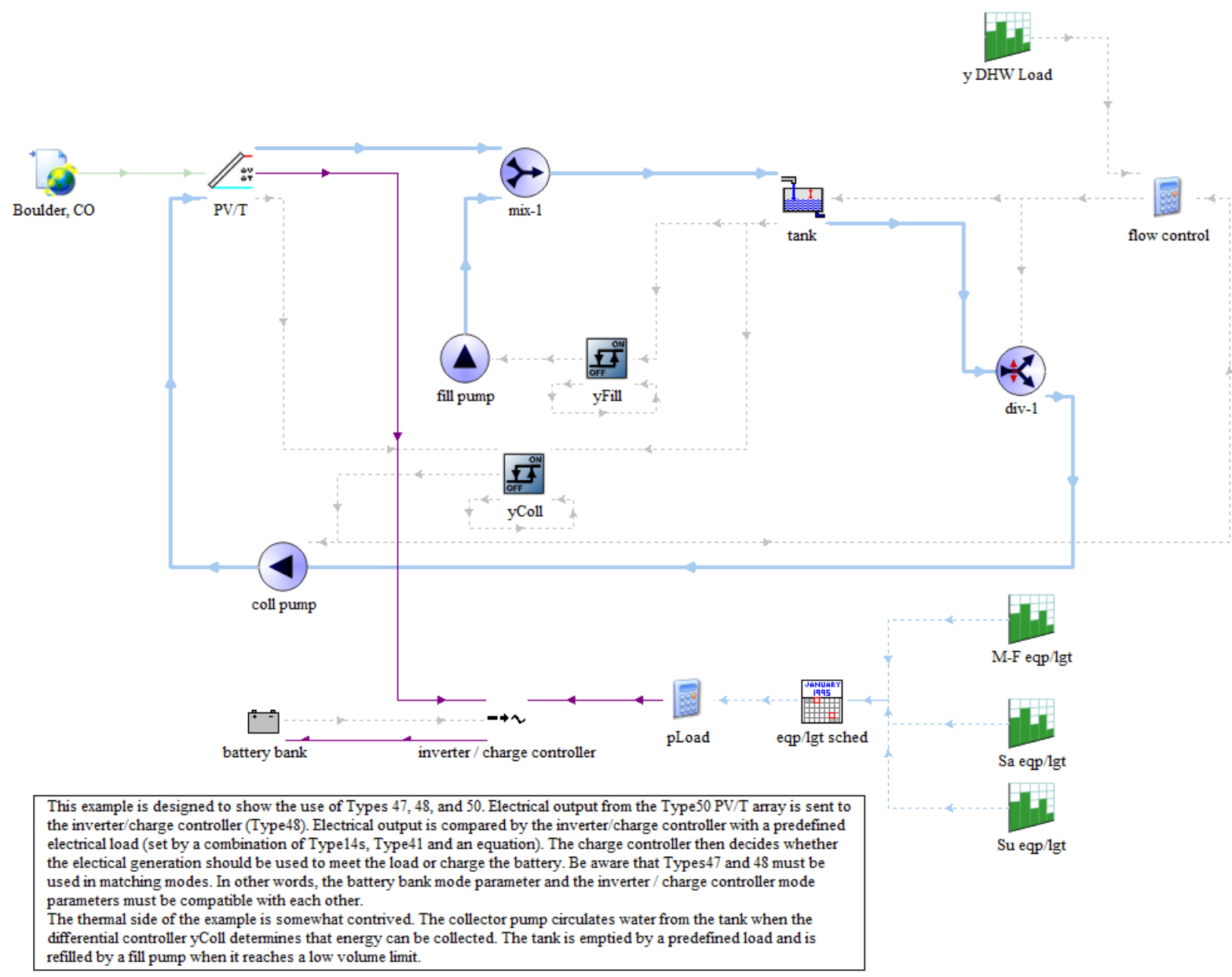

Figure 4. The PVT example model in TRNSYS [1]

This is a simple calculation, but the purpose of using the PVT example is to demonstrate the process of exchanging data between TRNSYS and Java. More complex calculations can be performed in a similar manner. A case study for assessing the performance of six residential energy management control algorithms through co-simulation of TRNSYS and MATLAB facilitated by Type277 is presented in [2], [4].

\subsection{Downloading JTRNSYS Project}

This section provides instructions for downloading a Java project that contains all the necessary folders and files used for loosely-coupled integration of TRNSYS and the Java environment. It is assumed that the 64-bit version of TRNSYS 18 has been installed in the C:ITRNSYS18 folder.

1. Download the zip file or clone JTRNSYS project from https://github.com/usnistgov/JTRNSYS.git;

2. Extract jtrnsys-master.zip or clone to your desired location, e.g., C:ljtrnsys-master;

3. In the jtrnsys-master folder: 
a. Navigate to the "TRNSYS 64bit" folder and copy the "Trnsys Socket Client" folder into the "C: $\mathrm{ITRNSYS18 \backslash Studio \backslash Proformas"} \mathrm{folder;}$

b. Navigate to the "TRNSYS 64bitlPVT Example" folder and copy the PVTType277.tpf file into the "C:ITRNSYS18\Examples\PVT" or into another desired folder such as "C:ITRNSYS18\MyProjects";

c. Navigate to the "TRNSYS 64bit।Type277DLLs\ReleaseDLL" and copy the Type277.dll file into the release folder in "C:\TRNSYS18\UserLib\ReleaseDLLs"; and

d. Navigate to the "TRNSYS 64bitlType277DLLs\DebugDLL" and copy Type277.dll file into the debug folder in "C:\TRNSYS18\UserLib\DebugDLLs".

To use the 32-bit version of Type277, follow step 3, but copy files from the "TRNSYS 32bit" folder instead of the "TRNSYS 64bit" folder. The 32-bit version of Type277 will work with both TRNSYS17 and the 32-bit version of TRNSYS18.

\subsection{Co-Simulation between TRNSYS and Java}

To perform co-simulation between TRNSYS and Java navigate to the jtrnsys-master folder and follow these instructions:

1. Double click the ServerUILaunch.bat file or type java -jar "LaunchServerUI.jar" in Windows command prompt to launch the GUI as shown in Figure 5; and

2. Double click the Start Server button to launch the server. Do not close the server, it must remain active to accept a client's connection. Note that this action may cause a windows firewall security alert, allow the process to proceed.

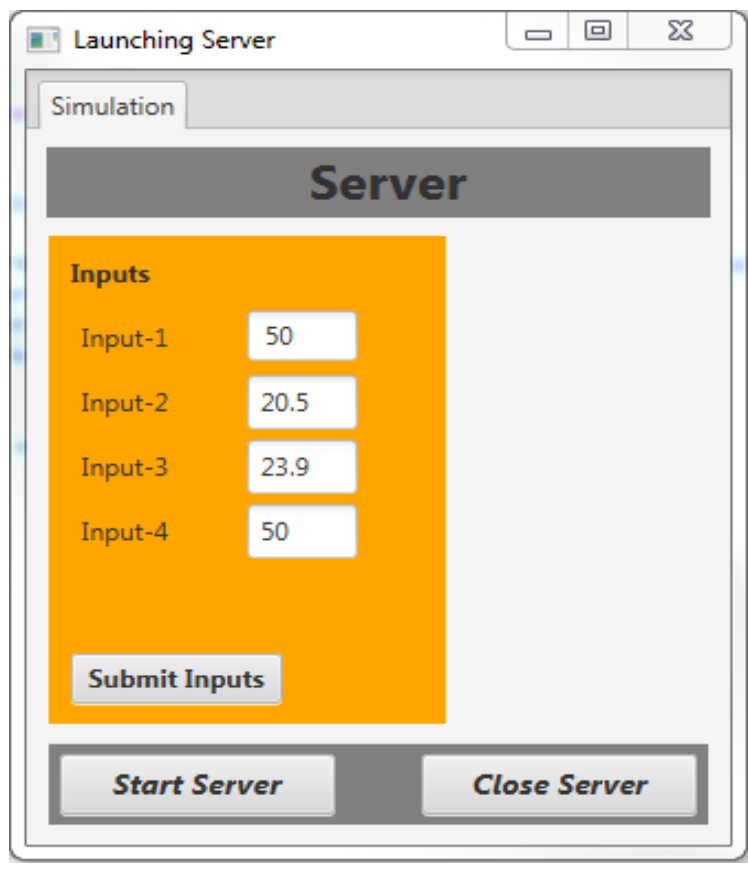

Figure 5. User interface for starting the server 
As can be seen from Figure 5, the Server GUI provides a simple interface for capturing user input data, starting the server, and finally terminating the server and closing the GUI. The four generic input fields are provided as a mechanism for users to submit their desired values. For example, users can set heating and cooling setpoints or define threshold limits for controlling humidity levels. These inputs are captured as text and parsed as the primitive type Double in ServerController class. The number of input fields can be extended as needed either graphically by manipulating the Server GUI or editing the JServer. fxml file included in the JTRNSYS project. To capture user inputs, double click the Submit Inputs button. In the current implementation, the data from the GUI is stored in an object of UI InputData class. A method, sendUIData () in ServerController class, is provided for sending the GUI input data through an instance of a Singleton object. Note that this is an additional functionality and not necessary to launch the server or communicate with TRNSYS.

After launching the server, it is ready to listen for a client connection on port 1345, which is used by Type 277 to communicate with the local server. The client is a TRNSYS simulation model that uses Type277. The port number (1345) was arbitrarily chosen, but it is hard-coded in Type277.

The next step is to simulate the modified version of the PVT project with Type277 as explained in the following section. Note that before simulating the TRNSYS model, the server must be running. In the absence of having an active server, TRNSYS will not throw an error if the model is simulated. Instead, the simulation will progress slowly, where the value for pLoad will be equal to zero.

\subsubsection{Simulating the PVT Model in TRNSYS}

The purpose of this section is to load the modified version of the PVT model (PVTType277) into the Simulation Studio and simulate it.

1. Open the 64-bit or 32-bit installation of Simulation Studio in TRNSYS18;

2. Select File $\rightarrow$ Open ... from the menu and navigate to the folder where the PVT example for Type277 was copied in Step b of Section 3.1;

3. Select the PVTType277.tpf file and click Open as shown in Figure 6. 


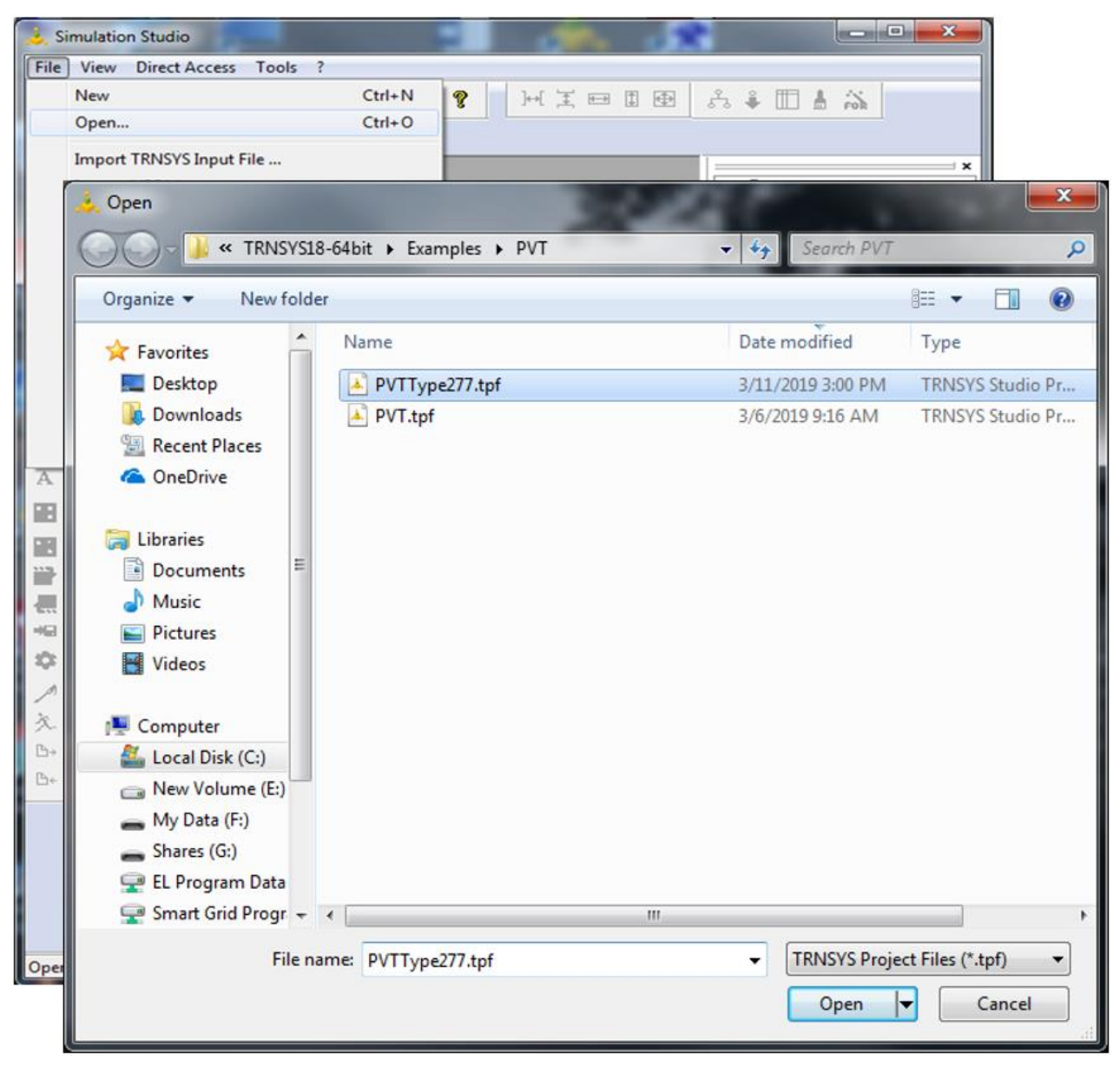

Figure 6. Open the PVTType 277 model

The PVT model with Type277, shown in Figure 7, is loaded into Simulation Studio. 


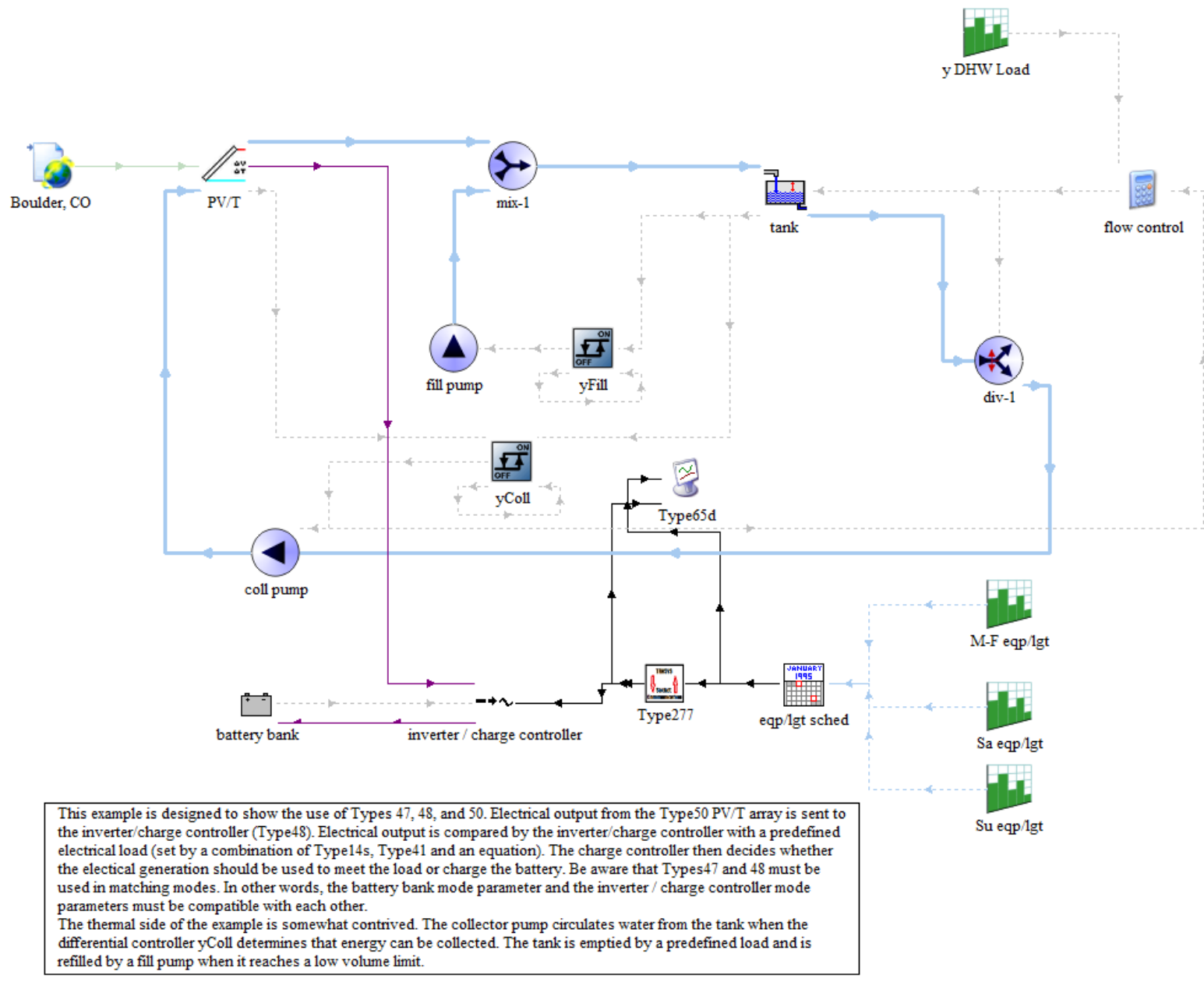

Figure 7. The PVT model with Type 277

A comparison of Figure 4 and Figure 7 shows that the Equation function for calculating pLoad in Figure 4 is replaced by the proforma for Type277. The input to Type 277 is fLoad, and the output is pLoad.

4. In Simulation Studio:

a. Navigate to the Calculate menu item and select Create input file from the dropdown menu as shown in Figure 8 and click $O K$; and

b. Run the PVTType277 example by selecting the Run button in Simulation Studio. This step launches the TRNEXE software, showing the simulation progress and values for pLoad calculated in Java. Select the Graph 1 to see the results of this simulation as shown in Figure 9. 


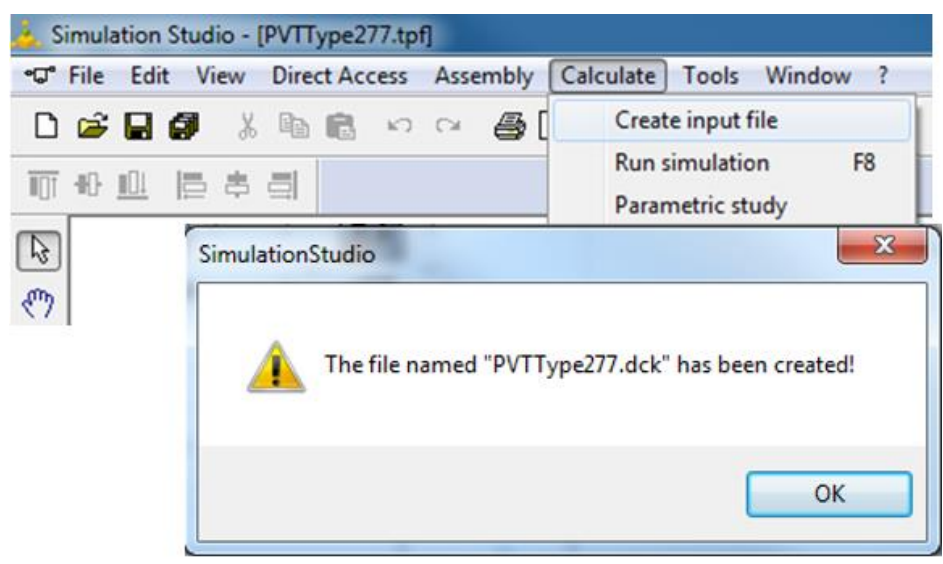

Figure 8. Create a TRNSYS input file for PVTType 277 model

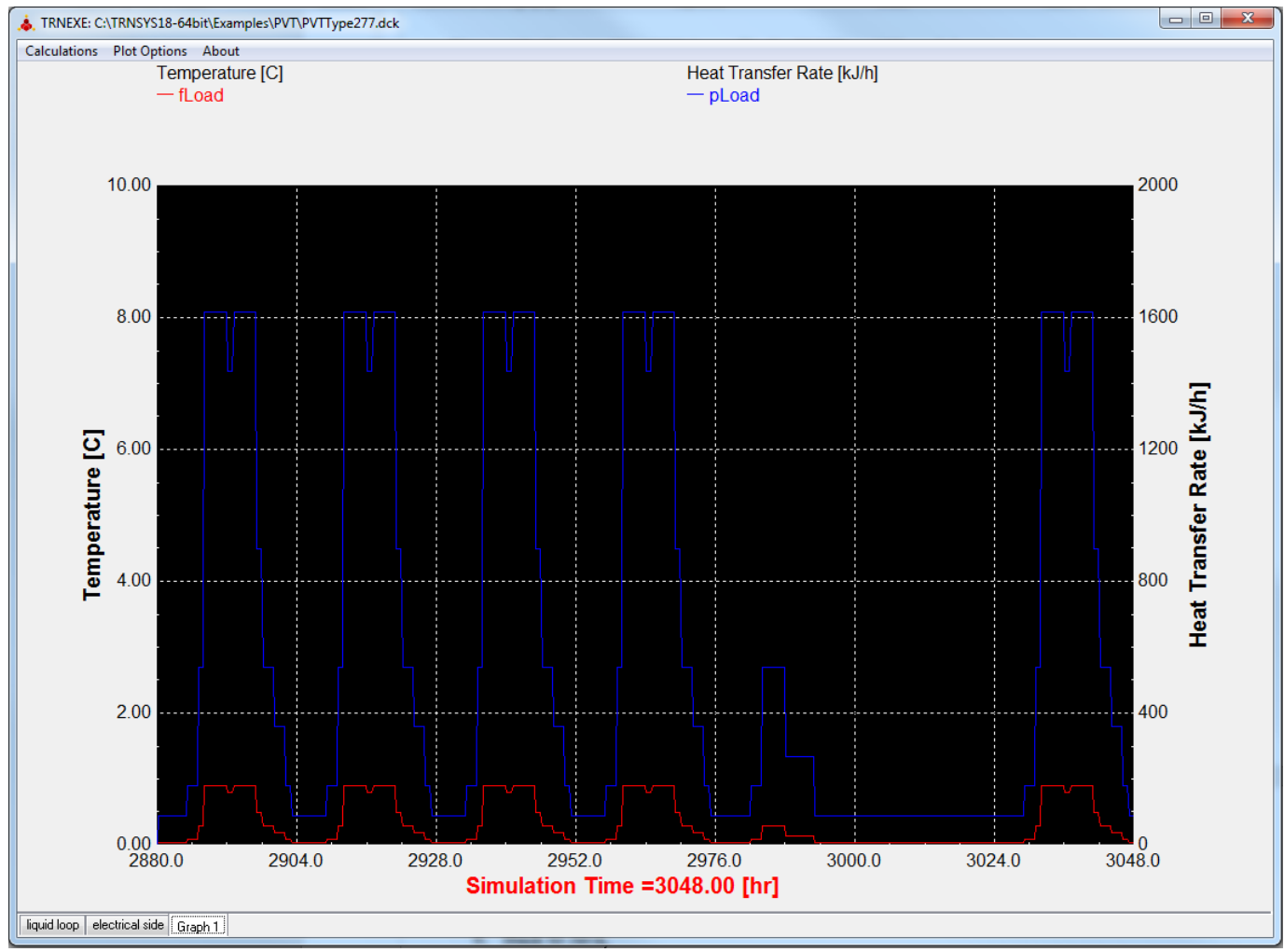

Figure 9. Result of simulating the PVTType277 example with the local server in Java

\section{Accessing Data and Modifying the Code}

The objective of this section is to describe the process for accessing data, modifying the code, and re-building the Java project. There are two main classes ServerRunnable and ServerRunnableMatlab, which provide access to the data and the ability to modify the code. In the ServerRunnable class, a user can access the data from TRNSYS and modify the code to meet their needs in the Java environment. Note that ServerRunnableMatlab 
is a modified version of the ServerRunnable class with additional functionality of connecting with a third-party software tool to perform advanced computation. To achieve the objective of this section, the Java project from Section 3.1 was imported into the Eclipse integrated development environment (IDE). Note that using Eclipse is not required to edit or view the content of the Java project. Users can choose a different integrated environment or none. The instructions for importing the Java project and adding third-party libraries into Eclipse are documented in Appendix.

\subsection{ServerRunnable Class}

As previously mentioned, the ServerUI class is responsible for launching the GUI, which creates an object of the ServerController class. The ServerController class creates an object of the ServerRunnable class as shown in the code snippet of Figure 10. These JAVA classes are in the JTRNSY project repository (JTRNSY\src) downloaded in Section 3.1.

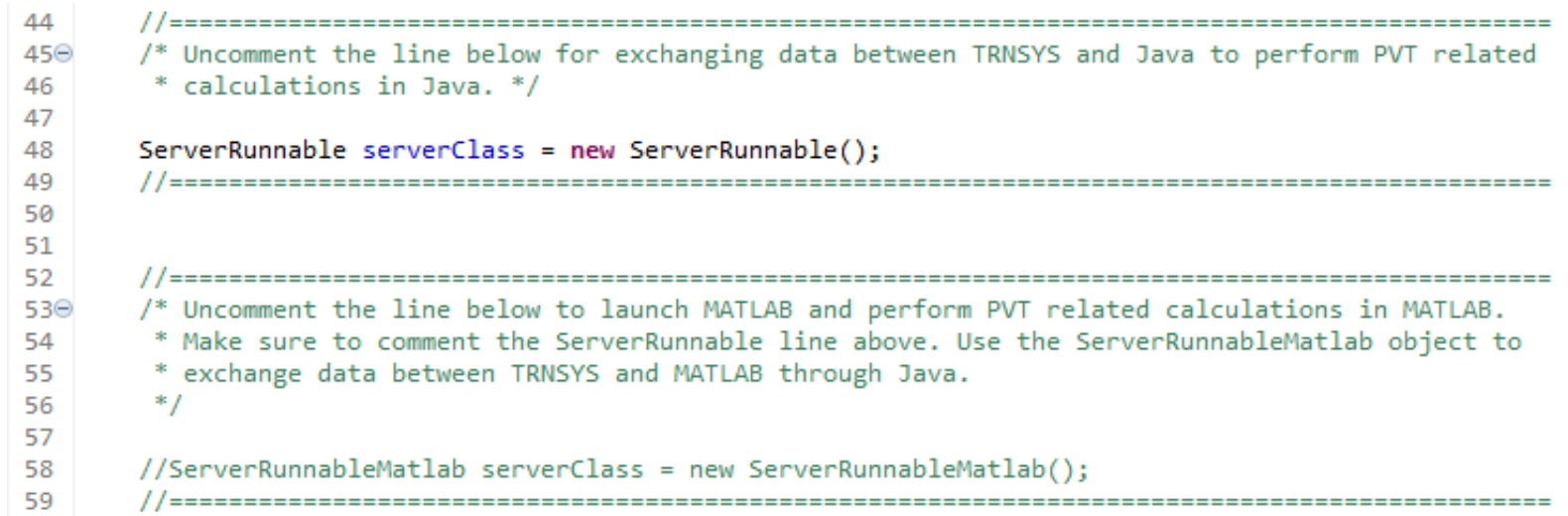

Figure 10. Create an object of ServerRunnable class for launching the server (see ServerController)

ServerRunnable is the main class for sending and receiving data to-and-from TRNSYS. It has three key functionalities: processing incoming data from TRNSYS, performing manipulation of incoming data, and sending the results back to TRNSYS. In the code snippet shown in Figure 11, the incoming data from TRNSYS (client) is read, deserialized, and parsed into a list and an array of the primitive type Double. This code is used to process incoming data and should function properly without modification. 


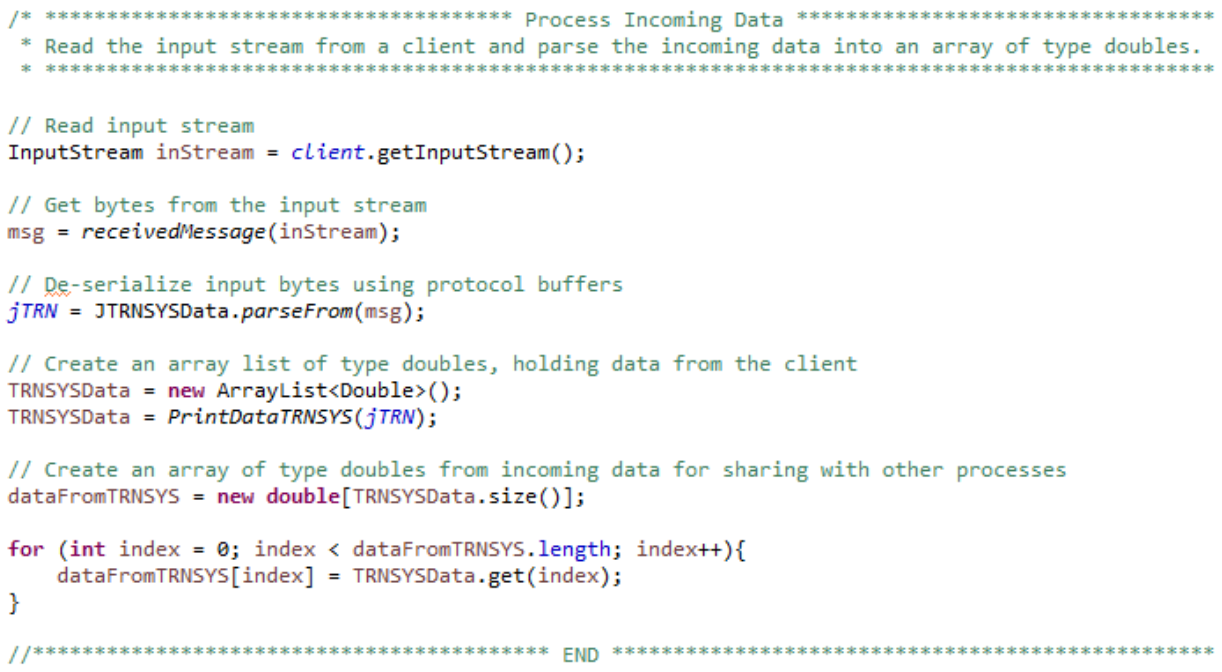

Figure 11. Code snippet for processing incoming data from TRNSYS (see ServerRunnable.java)

The array of the primitive type Double, dataFromTRNSYS, contains the data from TRNSYS. The number of entries in this array depends on the number of inputs connected to Type277as shown in Figure 3. In the modified PVT example fLoad is the only input to Type277; therefore, dataFromTRNSYS contains a single entry. Recall that fLoad is used to calculate pLoad. The dataFromTRNSYS is passed in as an input argument to the PVT ( ) method (Figure 12). The PVT () method (see ServerRunnable.java) returns an array of the primitive type Double, returnDataTo, which contains a single entry which is the calculated value of pLoad for a given value of fLoad. Use the "To Do" space shown in Figure 12 to access and perform manipulation of the data from TRNSYS.

\begin{tabular}{|l}
140 \\
141 \\
142 \\
143 \\
144 \\
145 \\
146 \\
147
\end{tabular}

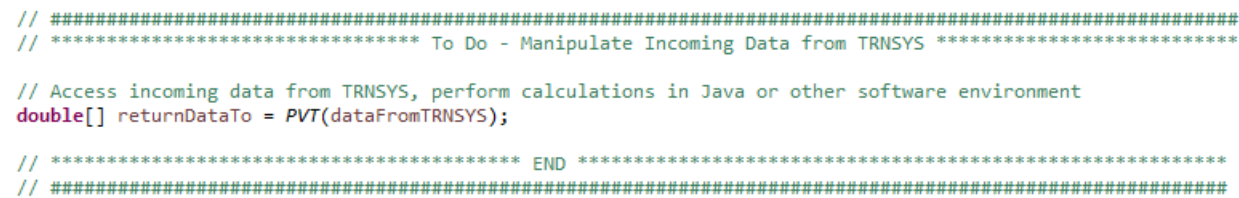

Figure 12. Access data from TRNSYS (see ServerRunnable.java)

The output of the PVT () method, returnDataTo, is passed as an input argument to the sendDataToTRNSYS () method (Figure 13). The sendDataToTRNSYS () is responsible for serializing and sending the data back to TRNSYS over the socket communication.

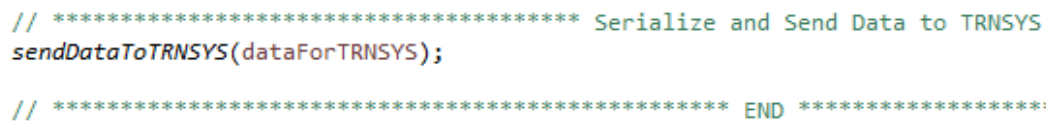

Figure 13. Sending serialized data back to TRNSYS (see ServerRunnable . java) 
An important feature of this client-server implementation is its ability to halt the simulation for debugging purposes. The TRNSYS simulation will not advance to the next timestep until it receives data from the server. Before using this functionality, the connection between the server and client must be established. In other words, before simulating the TRNSYS model, the server must be running. If a model is simulated without an active server, TRNSYS will not throw an error or display a warning. Instead, the simulation will progress slowly. To suspend the simulation, place a breakpoint in the ServerRunnable class and run the ServerUI class "JTRNSYSIsrclapplicationl" in debug mode.

\subsection{ServerRunnableMatlab Class}

In the previous section, the process for calculating pLoad in the Java environment was described. In this section, pLoad is calculated using MATLAB. Although TRNSYS has a MATLAB type, this section is used to demonstrate the functionality of the server communicating with a third-party software. The UML class diagram in Figure 14 shows the ServeruI, ServerController, and ServerRunnableMatlab that are included in JTRNSYSIsrc repository downloaded in Section 3.1. It describes the relationship of various classes used to support the loosely-coupled integration of TRNSYS and MATLAB via the server implemented in Java.

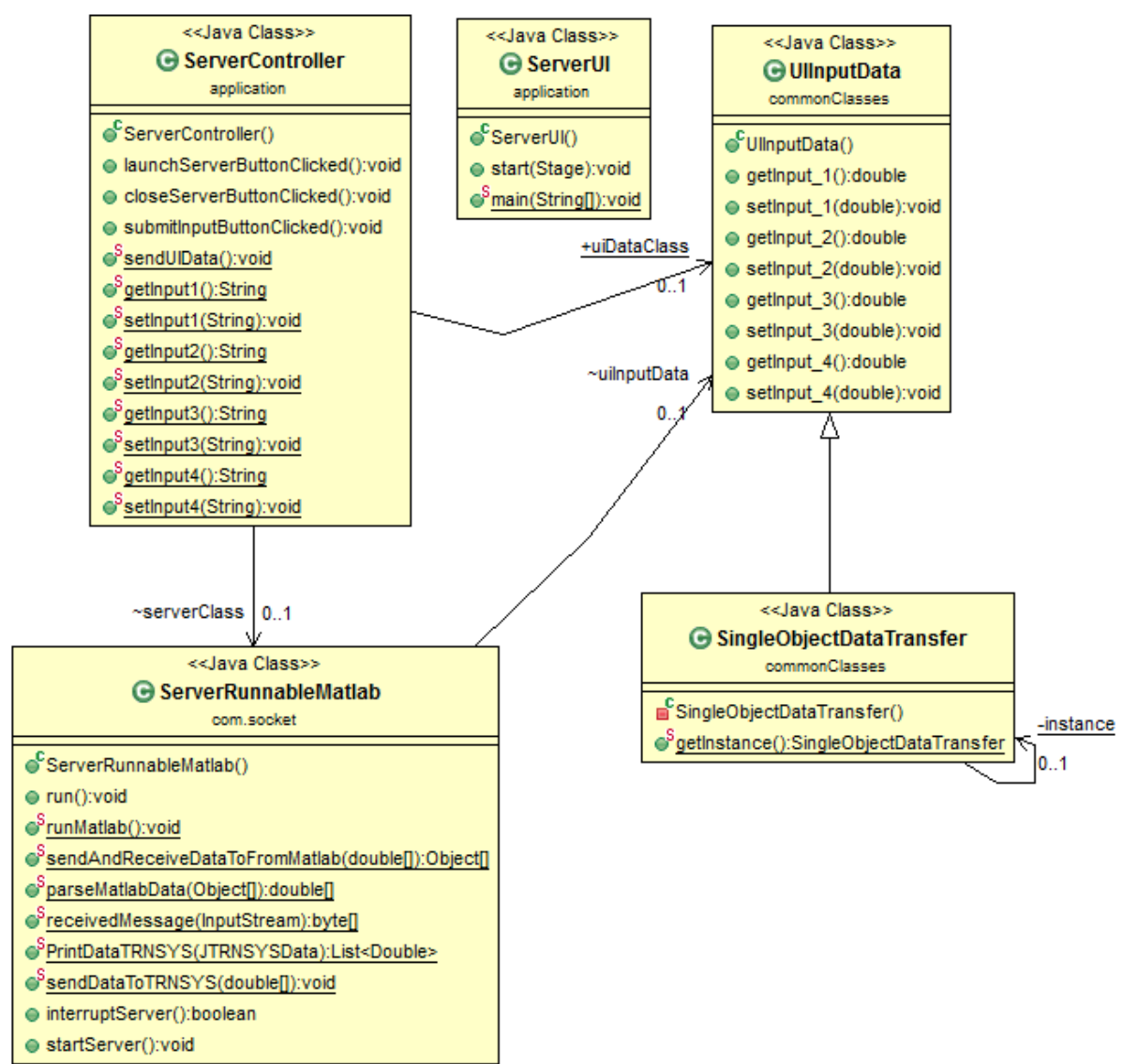

Figure 14. UML class diagram for loosely-coupling TRNSYS and MATLAB via the Java environment 
Like before, ServerUI is the main class for rendering the GUI and creating an instance of the ServerController. The ServerController class controls the functionality of the GUI and instantiates an object of the ServerRunnableMatlab class as shown in the code snippet of Figure 15. This change is accomplished by commenting out the ServerRunnable object on line 48 of Figure 15 and uncommenting the ServerRunnableMatlab object on line 58. Once the GUI is rendered (Figure 5), double clicking the Start Server button will launch the server and MATLAB.

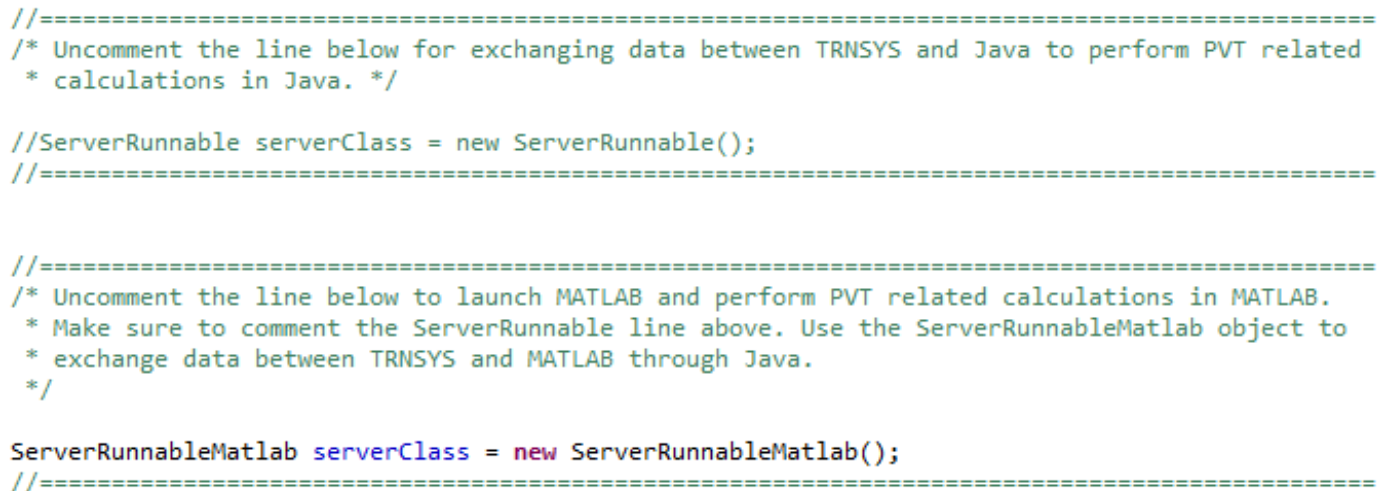

Figure 15. Create an object ServerRunnableMATLAB for exchanging data between TRNSYS and MATLAB

ServerRunnableMatlab is the main class for launching the server and facilitating the exchange of data between TRNSYS and MATLAB. It has three key functionalities: processing incoming data from TRNSYS, sending and receiving data to-and-from MATLAB to perform calculation, and finally sending the results back to TRNSYS. The ServerController class also instantiates an object of UIInputData class to capture the user inputs from the GUI and transfer them to other classes using a Singleton object.

In ServerRunnableMaltab class, processing the incoming data from TRNSYS is like the procedure described in Section 4.1 and shown in Figure 11. In the next section, the procedure for calculating pLoad in MATLAB is described.

\subsubsection{Performing Calculations in MATLAB}

After the server is launched and the MATLAB program is opened, right-click on "Matlab Files" folder and add it to the path as shown in Figure 16. There are two functions in this directory, the JavaMatlabDemoFunction(), and the PVTType277(). The JavaMatlabDemoFunction ( ) is the entry point to the MATLAB environment. The data from Java is communicated to MATLAB through the input argument of this function. The PVTType277 ( ) function in MATLAB computes pLoad for the given values of fLoad from TRNSYS. Complex calculations using MATLAB's analytical capabilities are performed in a similar manner. A case study for assessing the performance of six residential energy management control algorithms through co-simulation of TRNSYS and MATLAB facilitated by Type277 is presented in [2], [4]. 


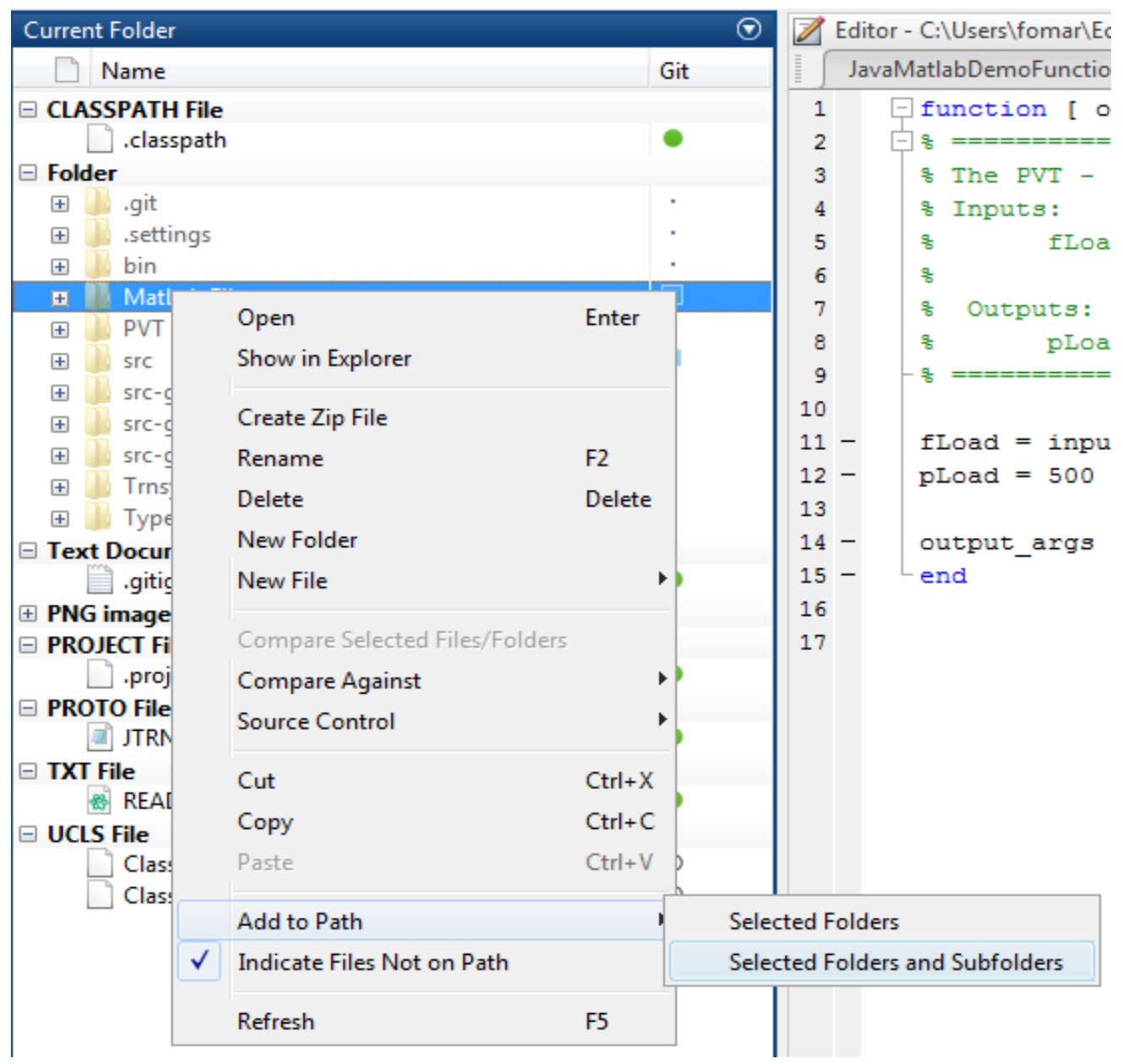

Figure 16. Adding Matlab Files folder to the path of MATLAB software

Recall that our intended task is to calculate pLoad in MATLAB; therefore, we need to send fLoad from TRNSYS to MATLAB to perform this calculation. Figure 17 shows the two methods that facilitate the indirect exchange of data between TRNSYS and MATLAB.

\begin{tabular}{|l}
159 \\
160 \\
161 \\
162 \\
163 \\
164 \\
165 \\
166 \\
167 \\
168 \\
169 \\
170 \\
171 \\
172 \\
\end{tabular}

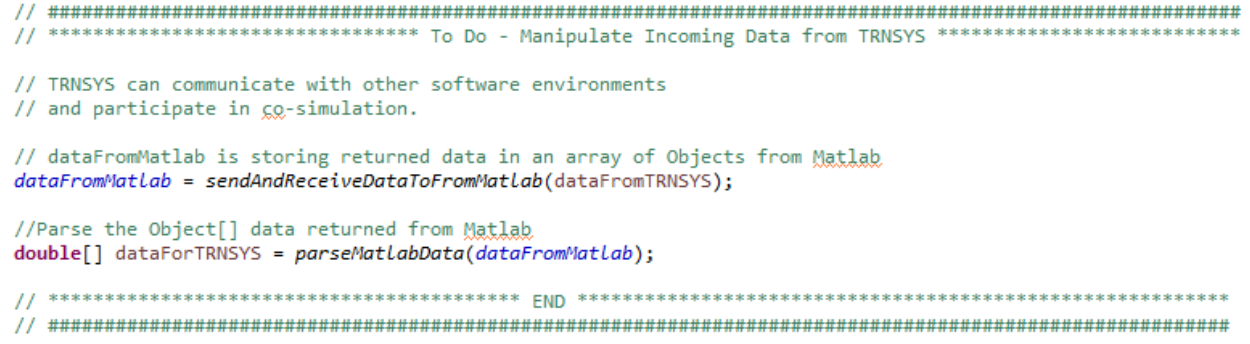

Figure 17. Methods for facilitating the exchange of data between TRNSYS and MATLAB

As can be seen from Figure 17, the datafromTRNSYS is passed as an argument to the sendAndReceiveDataToFromMatlab() method, which facilitates the exchange of 
data between Java and MATLAB through a proxy established by using the matlabcontrol library [5]. As shown in Figure 18, the proxy calls the JavaMatlabDemoFunction () and passes on the incoming data from TRNSYS. The output of this proxy function evaluation contains the returned values from MATLAB.

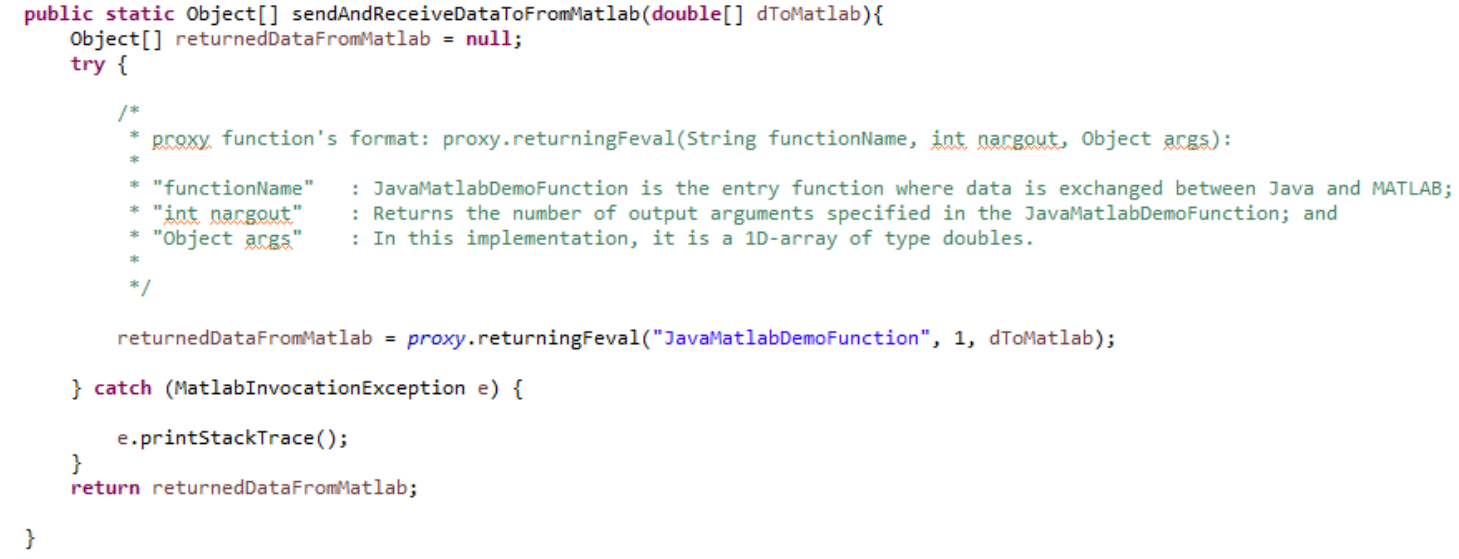

Figure 18. Proxy function call to send and receive data to-and-from MATLAB

The output of sendAndReceiveDataToFromMatlab () is an array of objects, which is the input argument to the parseMatlabData() method (Figure 17). The parseMatlabData () method extracts the result of calculations from the array of objects and returns an array of the primitive type Double, dataForTRNSYS. The sendDataToTRNSYS() method shown in Figure 19 takes dataForTRNSYS as an argument. It serializes the data and sends it to TRNSYS using socket communication.

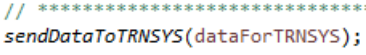

Figure 19. Method to serialize and return data to TRNSYS

As previously mentioned, an important feature of this client-server implementation is its ability to halt the simulation for debugging purposes. Before using this functionality, the connection between the server, client, and MATLAB must be established. In other words, before simulating the TRNSYS model, the server and MATLAB must be running. The path to the MATLAB functions must also be configured as described in Figure 16. To suspend the simulation, place a breakpoint in MATLAB. To pause the simulation in MATLAB, the ServerUI class "JTRNSYS Isrclapplicationl" does not need to be executed in debug mode. If a model is simulated with both the server and MATLAB running but the path to MATLAB functions are not configured as shown in Figure 16, Java will throw MatlabInvocationException, TRNSYS will display an error message, and MATLAB will display "Undefined function or variable JavaMatlabDemoFunction". The simulation will not run, and the user will need to relaunch the GUI and the server by double clicking the Start Server button. 
Figure 20 shows the PVTType277() function in MATLAB with a breakpoint which halted the TRNSYS simulation from stepping forward.

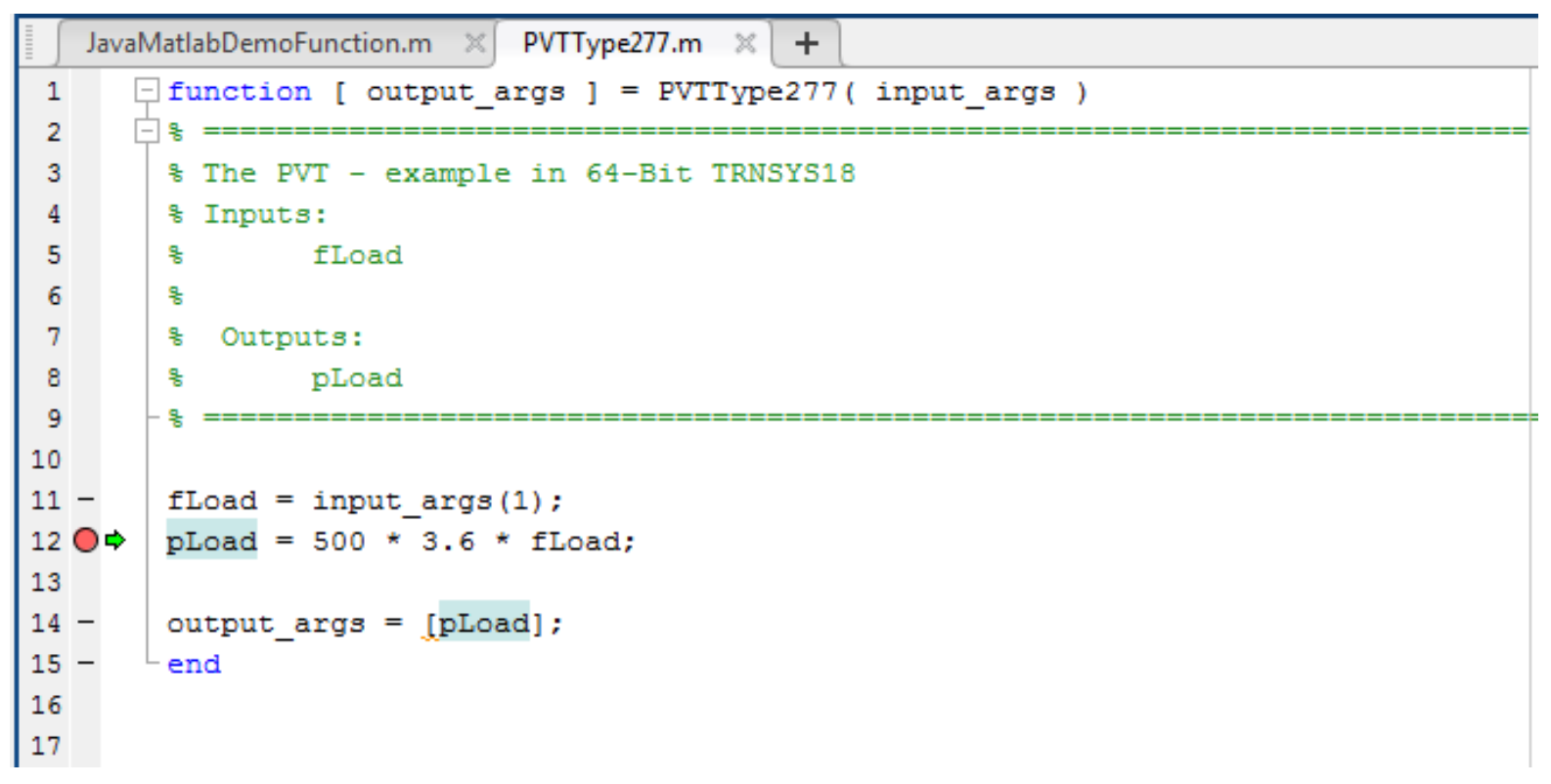

Figure 20. Debugging in MATLAB

\section{Conclusion}

An application of Type277 was successfully demonstrated for facilitating the loosely-coupled integration of a TRNSYS model with a Java environment and MATLAB. Type277 provides a simple and efficient mechanism for running a TRNSYS model in a co-simulation environment without changing the core functionality of the server for exchanging data. Type 277 is written in $\mathrm{C}++$ and compiled into 32-bit and 64-bit DLLs, supporting both versions of TRNSYS. It supports co-simulation of TRNSYS with multiple programming languages such as Java, C++, and Python. 


\section{Appendix - Importing JTRNSYS Project into Eclipse}

To import the Java project (jtrnsys-master) from Step 2 of Section 3.1 into an existing workspace or create a new workspace in Eclipse follow these instructions:

1. In Eclipse, choose File and select Import... from the drop-down menu. The Import wizard opens as shown in Figure 21;

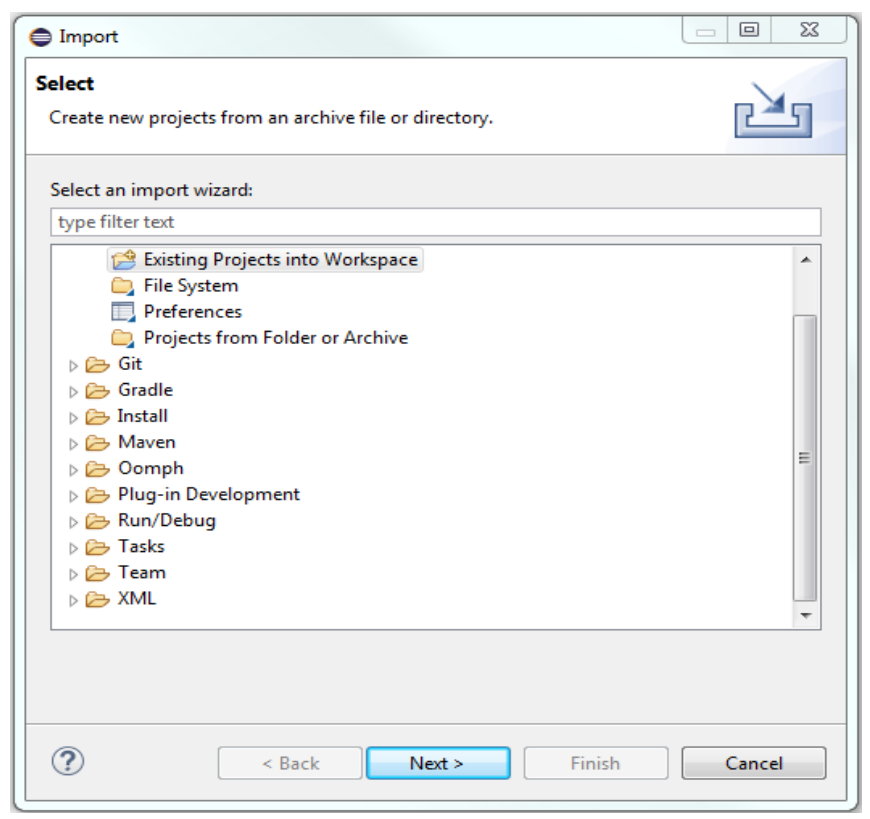

Figure 21. Eclipse import wizard

2. In the Import wizard, choose Existing Projects into Workspace and click Next;

3. In the Import Projects window, using the Browse button navigate to the directory where you extracted the zip folder jtrnsys-master;

4. Select the checkbox next to the Copy projects into workspace to make a copy of the project into your workspace as shown Figure 22; 


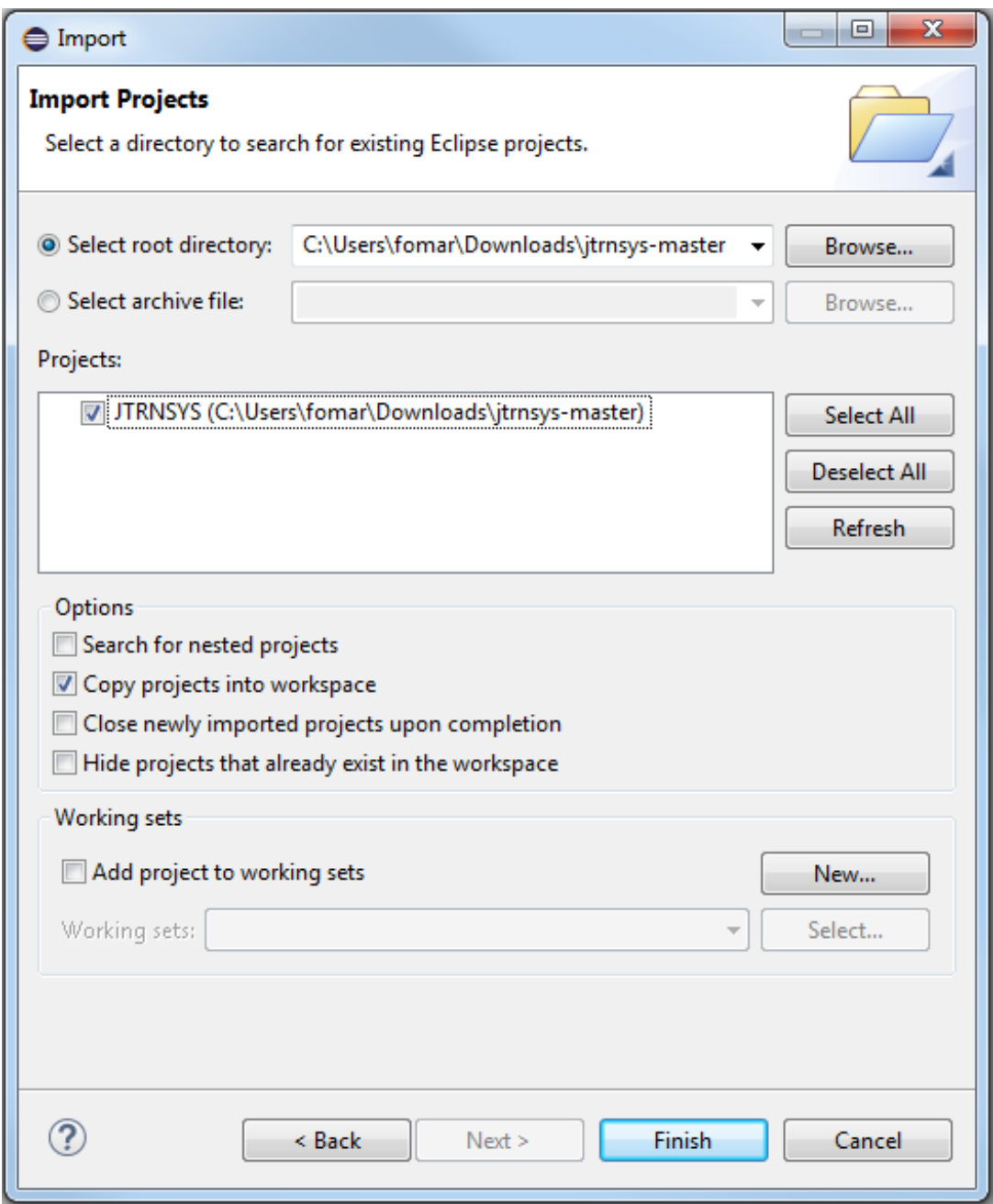

Figure 22. Import projects wizard 
5. Click Finish, this process will import JTRNSYS Java project into the workspace as shown in Figure 23.

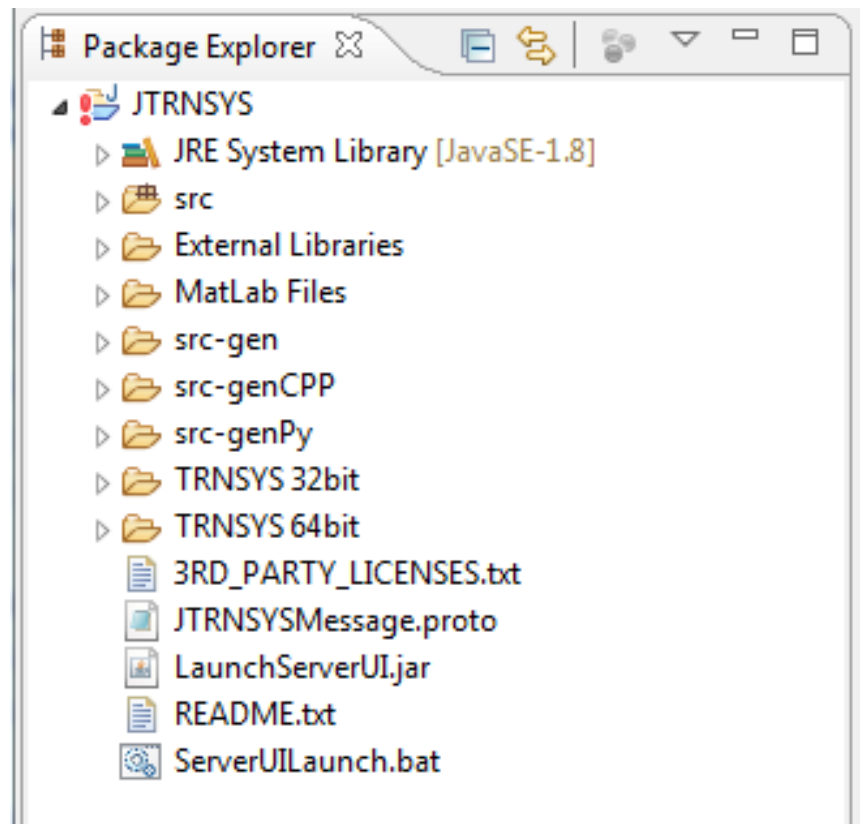

Figure 23. Project explorer in Eclipse

As can be seen from Figure 23, the Package Explorer (red exclamation mark) indicates that there are errors in the project. The errors are because of missing required protocol buffers, matlabcontrol, and JavaFX11 libraries. For convenience, a copy of these libraries is included in the JTRNSYS project under "jtrnsys-masterlExternal Libraries" folder, and a copy of the licenses for these libraries are reproduced in the $3^{\mathrm{RD}}$ _PARTY_LICENSES.txt file.

The following steps describe the process of adding these libraries to the JTRNSY project:

a. Right click on the JTRNSYS project and select Properties from the list as shown in Figure 24; 


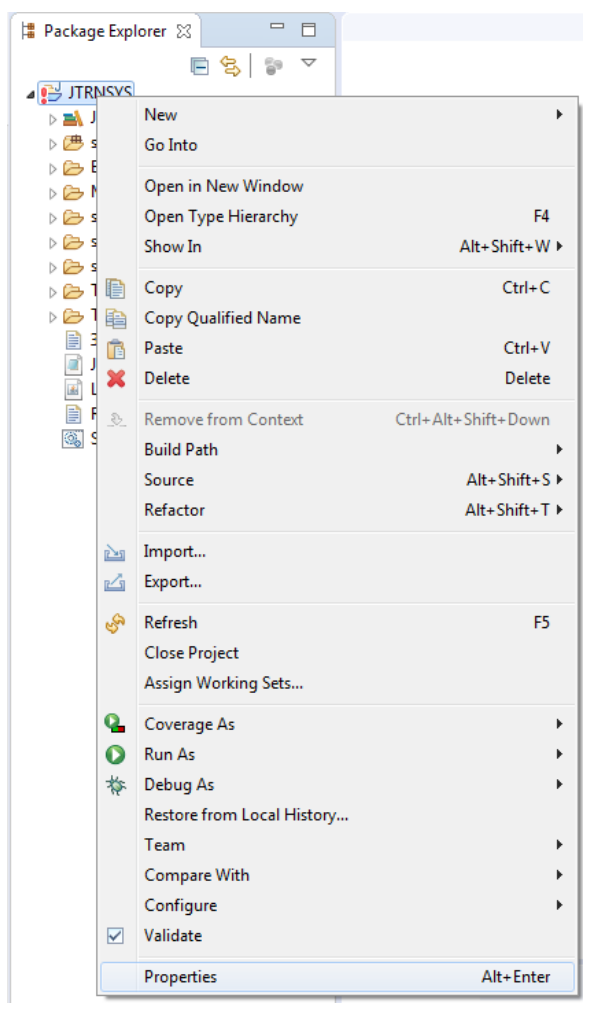

Figure 24. Opening properties window for JTRNSYS

b. In the Properties for JTRNSYS window, select Java Build Path and select the Libraries tab as shown in Figure 25. We first need to remove the references to these missing libraries and then add them from "jtrnsys-masterlExternal Libraries" folder downloaded in Section 3.1; 


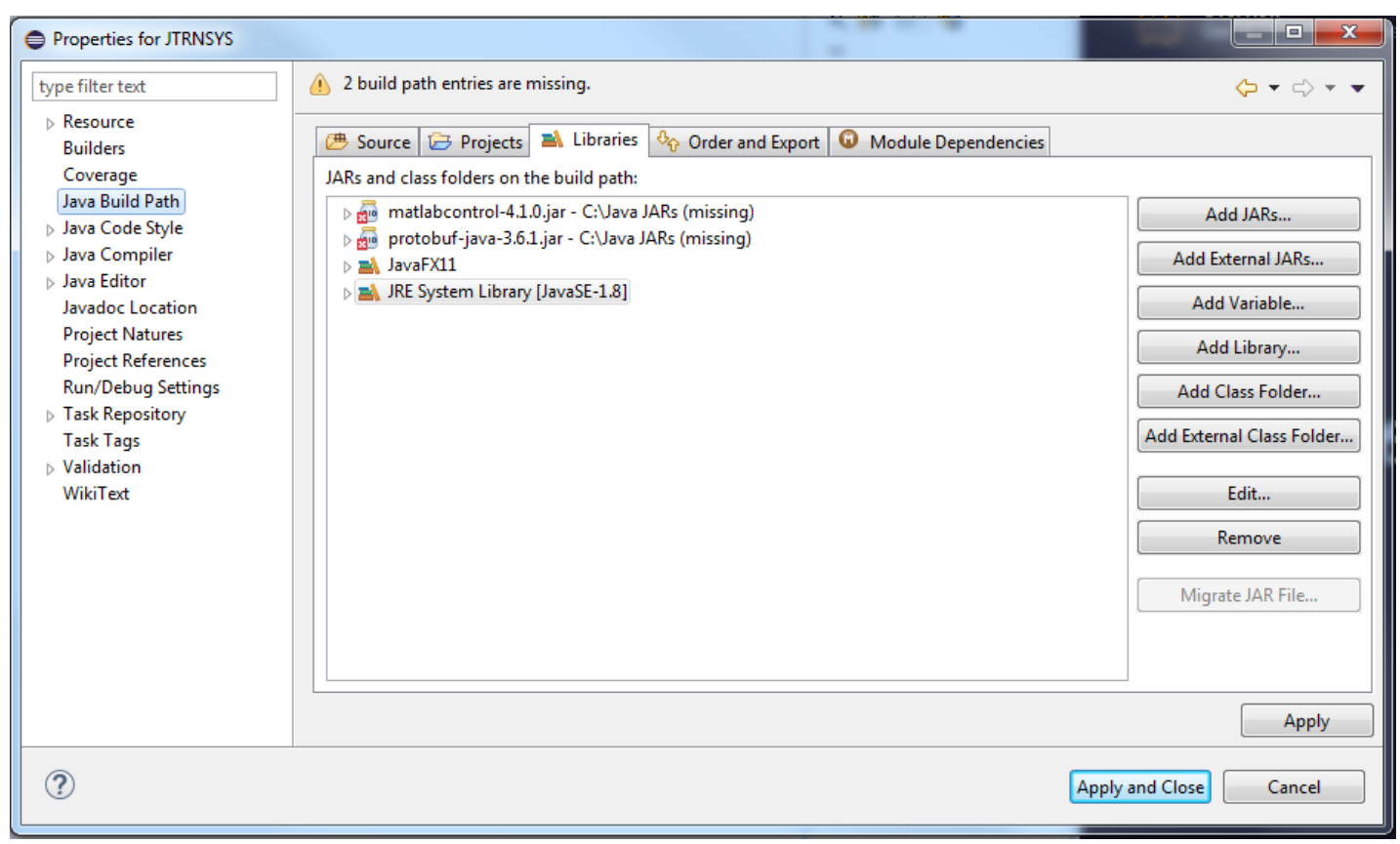

Figure 25. Editing Java build path for the missing libraries

c. To remove the missing references to matlabcontrol-4.1.0.jar, protobufjava-3.6.1.jar, as well as JavaFX11 libraries, select each library and click the Remove button as shown in Figure 25;

d. To add the missing libraries, click the Add External JARs button (Figure 25) and navigate to the "jtrnsys-masterlExternal Libraries" folder and select both matlabcontrol-4.1.0.jar and protobuf-java-3.6.1.jar files and click Open as shown in Figure 26. This step adds these two libraries on the build path for JTRNSYS project;

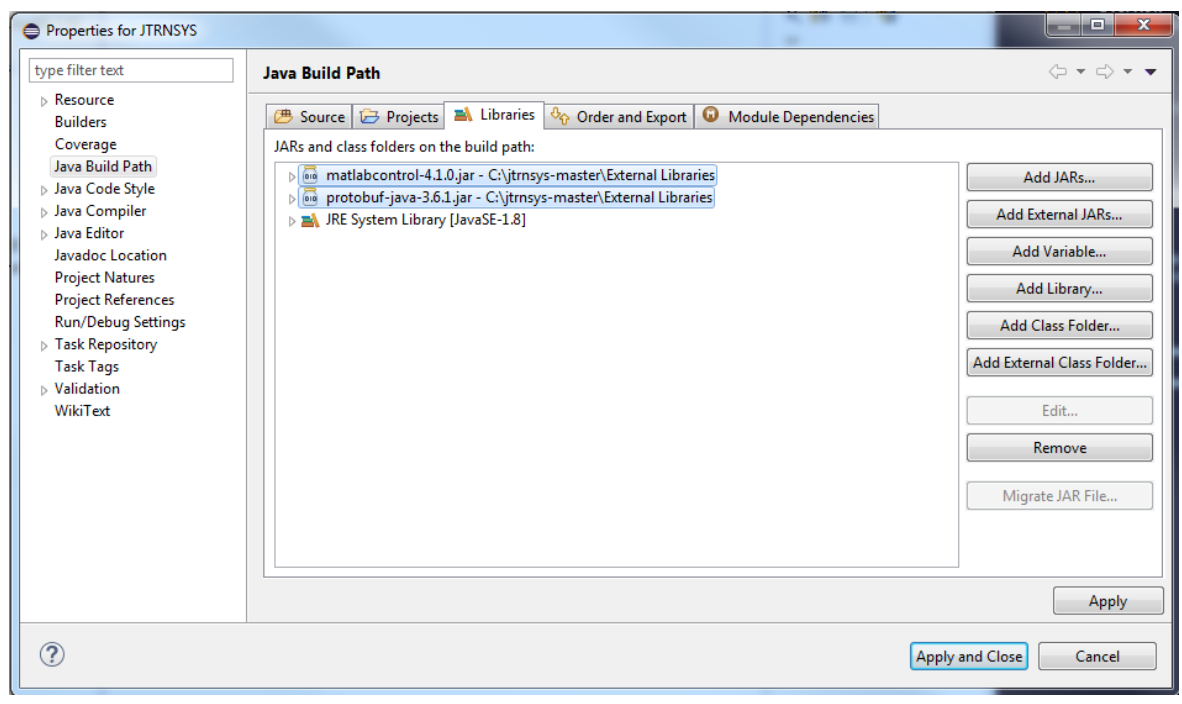

Figure 26. Adding third-party libraries to the build path for JTRNSYS project 
To add JavaFX $11^{1}$ library, click the Add Library button as shown in Figure 25.

e. In the Add Library window (Figure 27) select the User Library type and click Next;

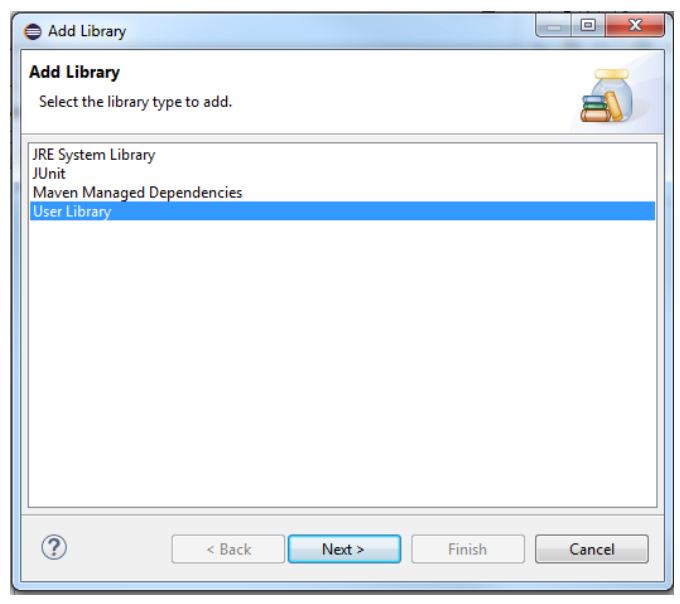

Figure 27. Adding a user library to the JTRNSYS project

f. In the Add Library window shown in Figure 28, click the User Libraries button to open the Preferences (Filtered) window, then click the New button to open the New User Library window. In the New User Library window, create a name for the new library like JavaFX11 or any other desired name, and click OK;

\footnotetext{
${ }^{1}$ The instructions for adding JavaFX 11 to the Eclipse IDE was obtained from https://openjfx.io/openjfx-docs/
} 


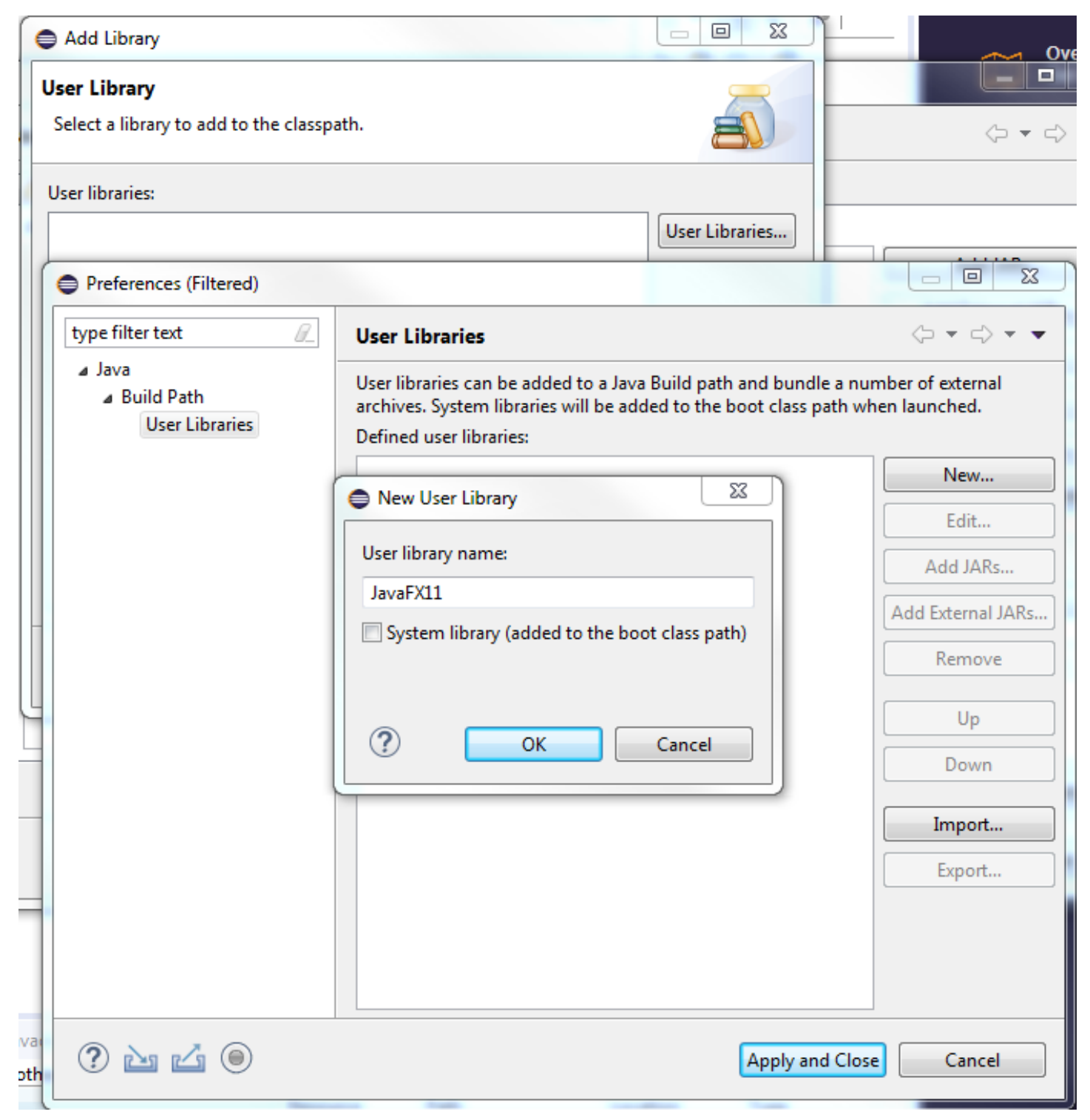

Figure 28. Adding a new JavaFX 11 user library to the JTRNSYS project

g. In Preferences (Filtered) window, click the Add External JARs (Figure 28) button and navigate to "C: Ijtrnsys-masterlExternal LibrariesljavafX-sdk-11.0.2 لlib". Select all jar files as shown in Figure 29 and click Open; 


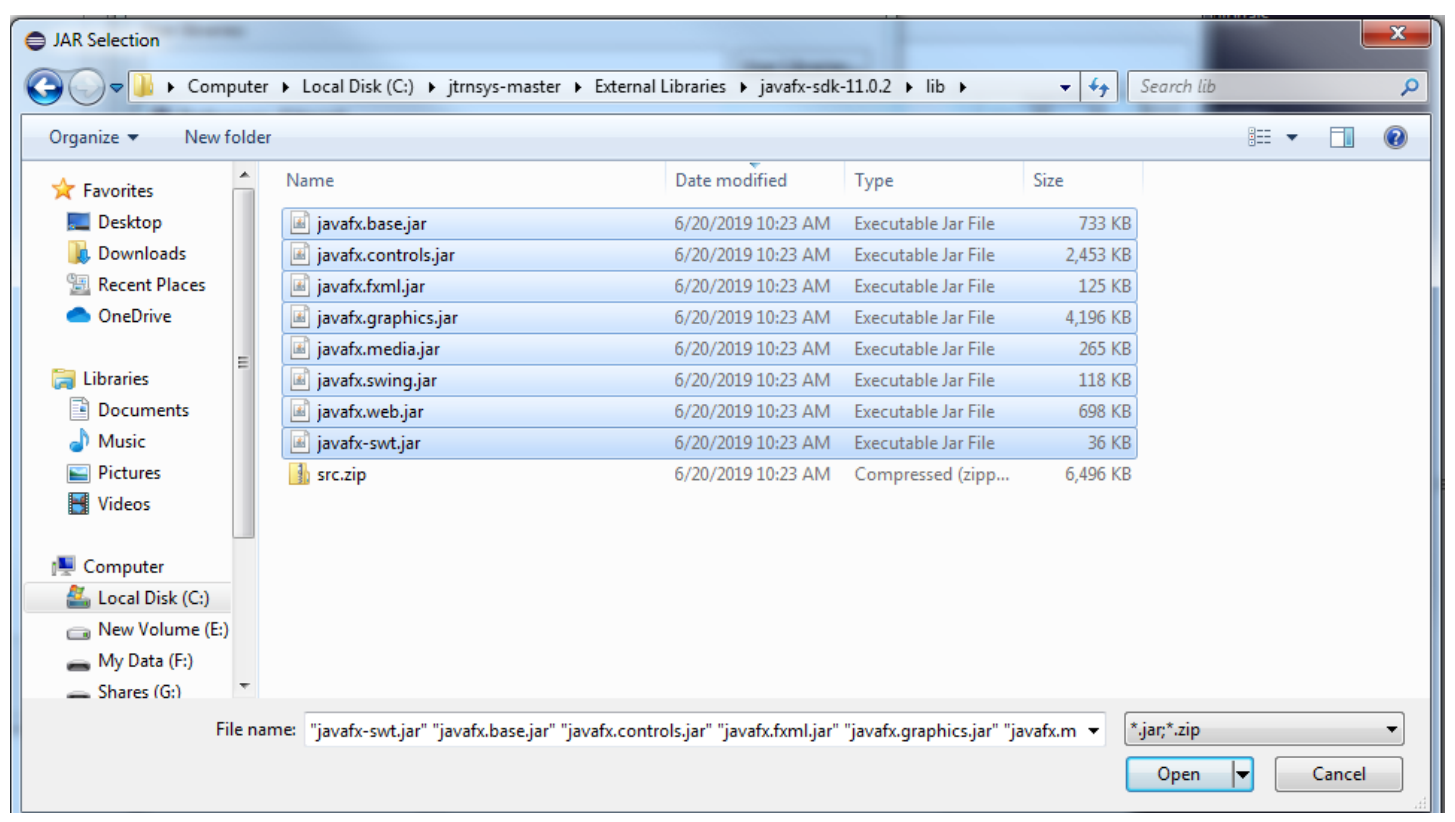

Figure 29. Selecting JavaFX 11 jar files

The procedure in Step 7 creates a JavaFX 11 user library as shown in Figure 30 that can be added to the Java build path for JTRNSYS project.

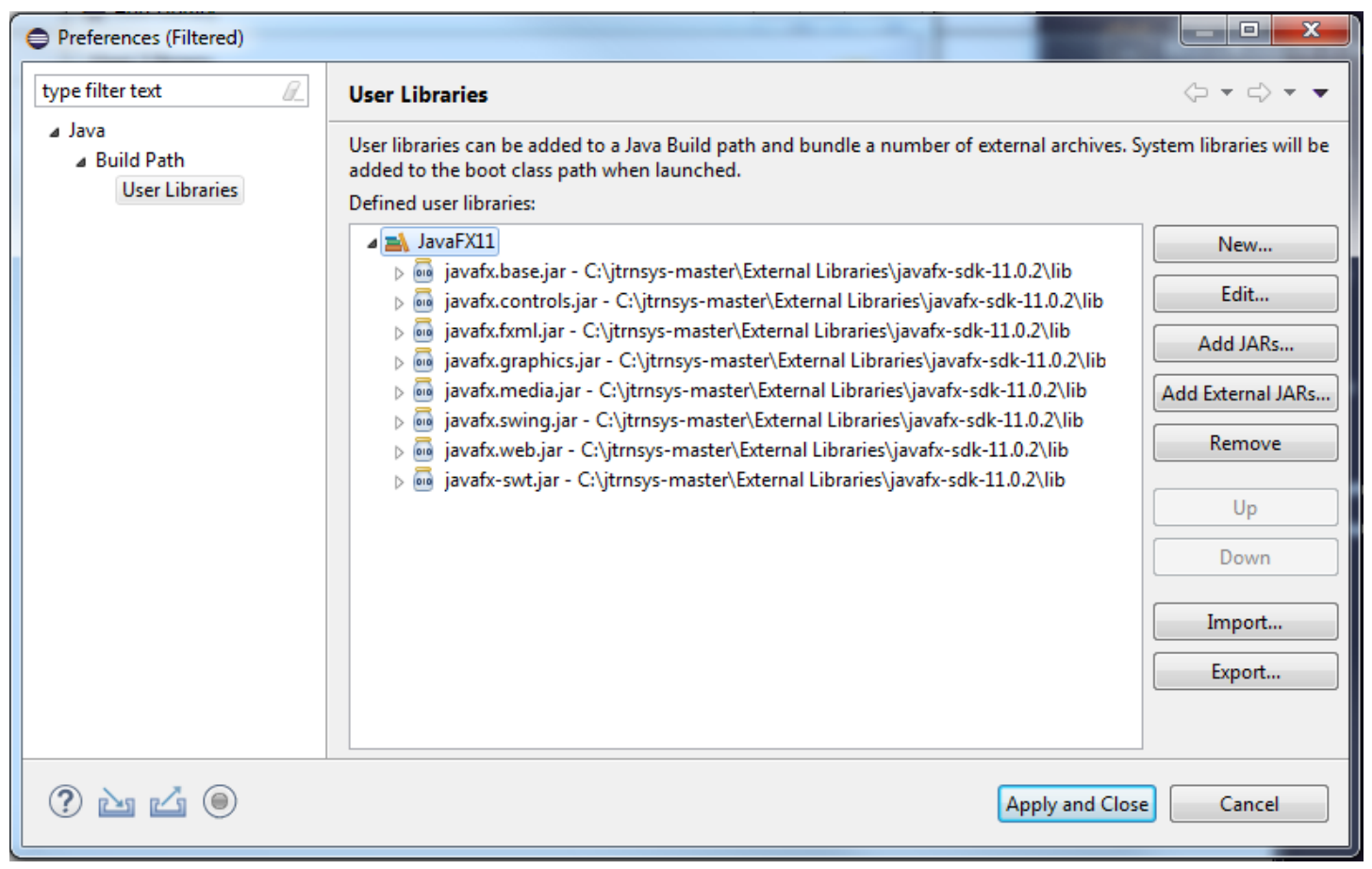

Figure 30. JavaFX 11 user library

h. Click Apply and Close button to close the Preferences (Filtered) window, and then click the Finish button to close the Add Library window (Figure 31); 


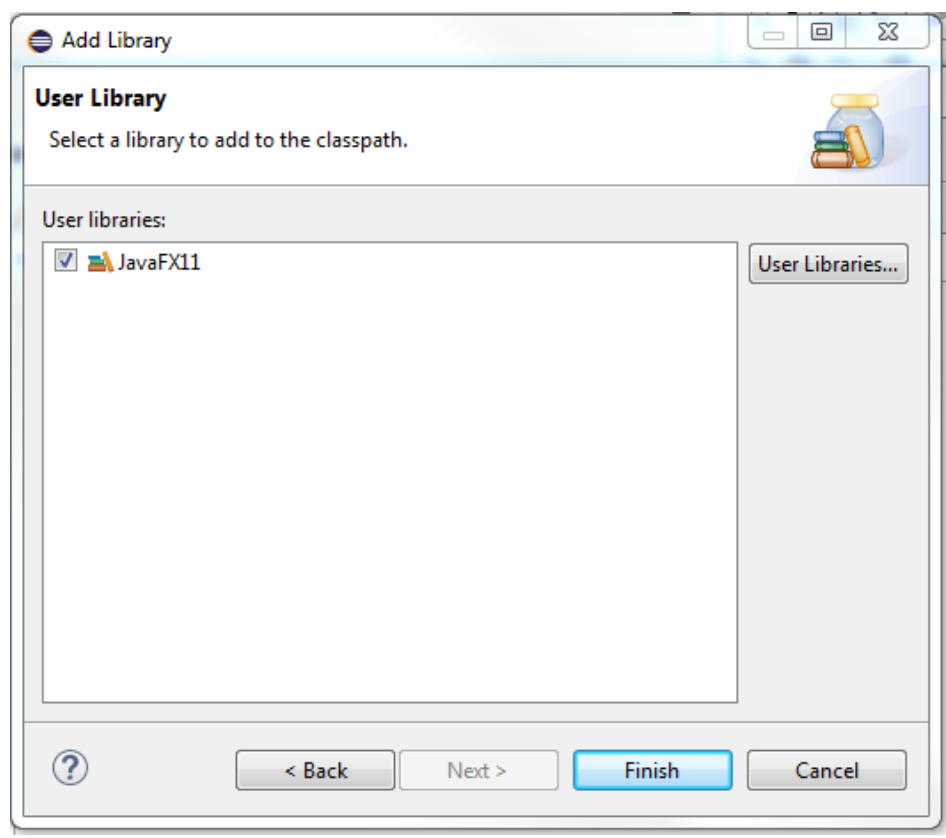

Figure 31. Close the Add Library window

As shown in Figure 32, the JavaFX11 user library has been added to the build path for JTRNSYS project. Click the Apply and Close button to exit the Properties for JTRNSYS window.

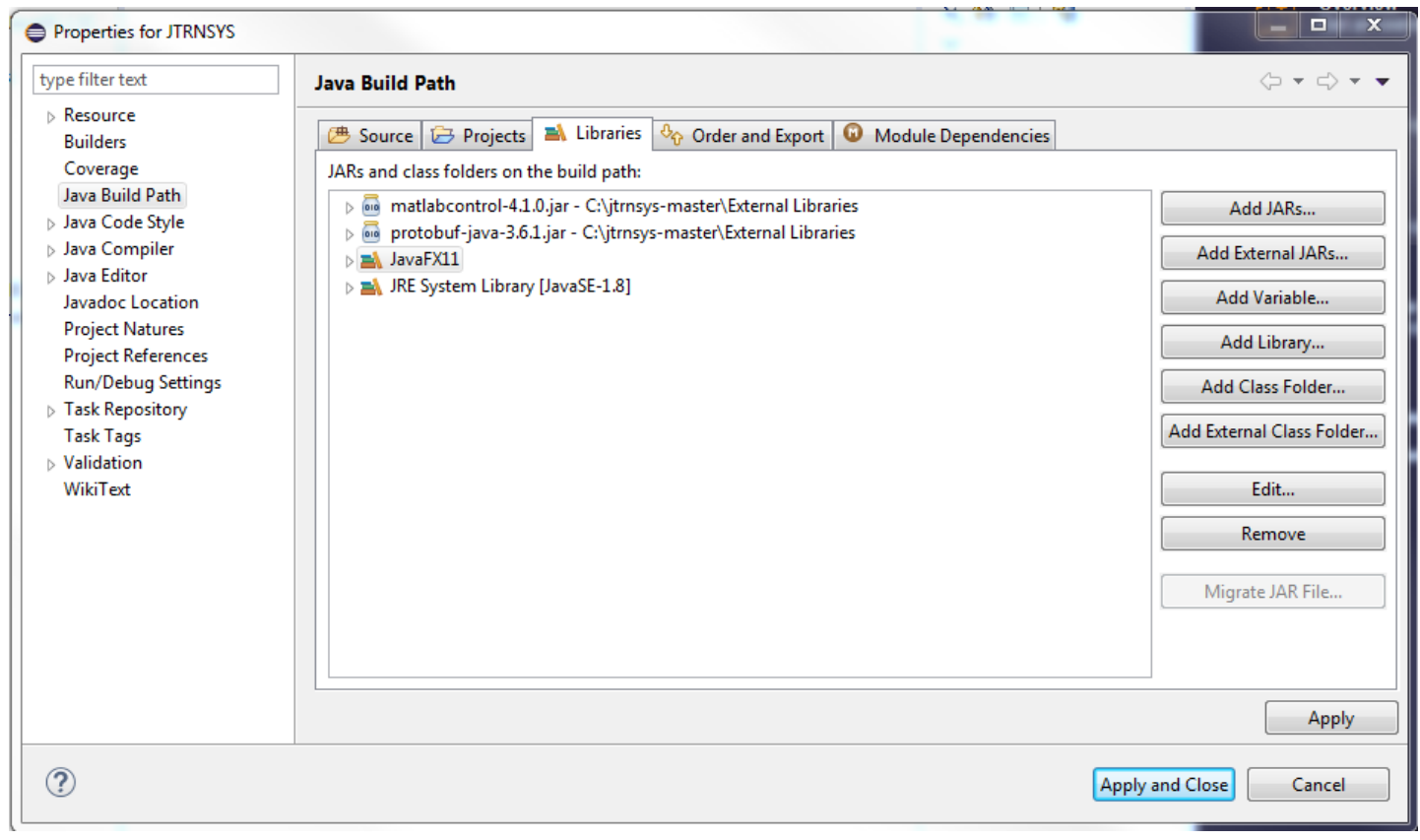

Figure 32. Added external libraries to the build path of the JTRNSYS project

The instructions in Step a through $\mathrm{h}$ eliminated the errors caused by missing the required external libraries. To launch the Server GUI from Eclipse (as shown in Figure 5), navigate to 
the "JTRNSYS/src/application" and run the ServerUI. java class as a Java Application. After the Server GUI is activated, follow the instructions in Section 3.2 to launch the server and start communicating with a simulation model in TRNSYS.

To access the data and modify the code, navigate to "JTRNSYS/src/com.socket" and open ServerRunnable.java or ServerRunnableMatlab.java class and follow the instructions provided in Section 4. 


\section{References}

[1] S. A. Klein et al, "TRNSYS 17: A Transient System Simulation program, Solar Energy Laboratory, University of Wisconsin, Madison, USA," Trnsys, 2010.

[2] F. Omar, "A Residential Energy Control Algorithm Assessment Tool for Smart Grid : Multi-Criteria Decision Making Using the Analytical Hierarchy Process," Ph.D. Dissertation, 2019. [Online]. Available: https://doi.org/10.18130/v3-d6fh-nj31.

[3] Google, "Protocol Buffers | Google Developers," 2008. [Online]. Available: https://developers.google.com/protocol-buffers/. [Accessed: 10-Sep-2014].

[4] F. Omar, S. T. Bushby, and R. D. Williams, "Assessing the Performance of Residential Energy Management Control Algorithms: Muti-Criteria Decision Making Using the Analytical Hierarchy Process," NIST TN 2017, 2018.

[5] Google product, "Google Code Archive - Long-term storage for Google Code Project Hosting.," 2013. [Online]. Available:

https://code.google.com/archive/p/matlabcontrol/. [Accessed: 17-Sep-2014]. 